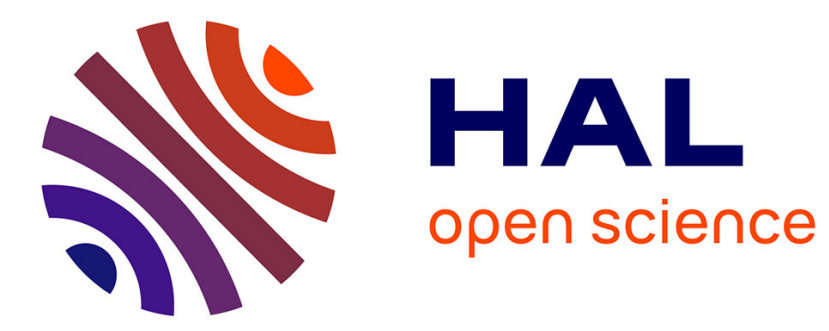

\title{
Nitric oxide (NO) photo-release in a series of ruthenium-nitrosyl complexes: new experimental insights in the search for a comprehensive mechanism
}

Max Roose, Marine Tassé, Pascal G. Lacroix, Isabelle Malfant

\section{- To cite this version:}

Max Roose, Marine Tassé, Pascal G. Lacroix, Isabelle Malfant. Nitric oxide (NO) photo-release in a series of ruthenium-nitrosyl complexes: new experimental insights in the search for a comprehensive mechanism. New Journal of Chemistry, 2019, 43 (2), pp.755-767. 10.1039/c8nj03907k . hal-02140608

\section{HAL Id: hal-02140608 \\ https://hal.science/hal-02140608}

Submitted on 4 Nov 2020

HAL is a multi-disciplinary open access archive for the deposit and dissemination of scientific research documents, whether they are published or not. The documents may come from teaching and research institutions in France or abroad, or from public or private research centers.
L'archive ouverte pluridisciplinaire HAL, est destinée au dépôt et à la diffusion de documents scientifiques de niveau recherche, publiés ou non, émanant des établissements d'enseignement et de recherche français ou étrangers, des laboratoires publics ou privés. 


\title{
Nitric oxide (NO) photo-release in a series of ruthenium-nitrosyl complexes: new experimental insights in the search of a comprehensive mechanism
}

\author{
Max Roose, ${ }^{\mathrm{a}}$ Marine Tassé, ${ }^{\mathrm{a}}$ Pascal G. Lacroix, ${ }^{{ }^{\mathrm{a}}}$ and Isabelle Malfant ${ }^{*^{\mathrm{a}}}$ \\ ${ }^{a}$ CNRS, LCC (Laboratoire de Chimie de Coordination), 205 route de Narbonne, 31077 Toulouse, \\ France.
}

*corresponding authors. E-mail : pascal.lacroix@1cc-toulouse.fr ; isabelle.malfant@1cc-toulouse.fr

\begin{abstract}
A series of four ruthenium (II) complexes built from the $\left[\mathrm{Ru}(\text { terpy)(bipy)(NO) }]^{3+}\right.$ core (terpy is the $2,2^{\prime}: 6^{\prime}, 2^{\prime \prime}$-terpyridine and bipy is the 2,2'-bipyridine) is investigated. They differ by the presence of zero, one, two, or three 4'-(4-methoxyphenyl) (MP) electron donor substituents introduced at different positions on the pyridine fragments to increase the intramolecular charge transfer capabilities towards the strongly withdrawing nitrosyl (NO) ligand. The UV-visible spectra reflect the presence and position of the MP substituents on the complexes. In the case of species containing the 4'-(MP)-terpy ligand, a low-lying transition is identified as arising from a 4'-(MP)-terpy to $\mathrm{Ru}(\mathrm{NO})$ intramolecular charge transfer, which is further confirmed by a TD-DFT analysis. Irradiations performed at $\lambda=436 \mathrm{~nm}$ on this isolated transition on different complexes lead to quantum yields of NO photo-release equal to 0.002 and 0.011 , in a ratio of 6 (instead of 1), which allows concluding non-ambiguously that a single electron transition cannot account for the NO release mechanism.
\end{abstract}




\section{Introduction}

Nitric oxide (NO·) has been recognized to possess many biological functions such as blood pressure regulation, stimulation of immune response, neurotransmission and cytotoxic activity in tumor cells by apoptosis. ${ }^{1,2}$ These numerous potential therapeutic actions have prompted research aimed at developing efficient $\mathrm{NO} \cdot$ donor molecules. Among them, ruthenium-nitrosyl [Ru(NO)] complexes provide particularly appealing candidates in relation with their generally low toxicity, good chemical stability, and moreover their capability to release NO $\cdot$ under light irradiation in the $\lambda=300-600 \mathrm{~nm}$ domain, exclusively, ${ }^{3,4}$ taking advantage of the noninvasive and highly controllable characteristics of light, according to the following equation:

$$
\left[R u^{I I}\left(N O^{+}\right)\right]+\text {solvent } \stackrel{h v}{\rightarrow}\left[R u^{I I I}(\text { solvent })\right]+N O
$$

NO is a classic non-innocent ligand and metal-nitrosyls can have different electronic structure descriptions depending on their coordination environment. In the complexes here under investigation, the total number of electrons present in the ruthenium $3 d$ orbitals and the $\pi^{*}$ orbitals of the nitrosyl ligand is equal to 6 , which is written $\{\mathrm{RuNO}\}^{6}$ in the Enemark and Feltham notation which avoids the assignment of a formal oxidation state of the metal and a charge on the ligand. ${ }^{5,6}$ However, it is reported that almost any $\{\mathrm{RuNO}\}^{6}$ electronic configurations correspond to the formal $\mathrm{Ru}^{\mathrm{II}} \mathrm{NO}^{+}$ electronic structure, which is EPR silent and in which the nitrosyl is regarded as a cation. ${ }^{7}$ The presence of this resulting diamagnetic singlet ground state has been further supported by several computational studies. ${ }^{8-11}$ Under these assumptions, the photo-release of the NO radical depicted in equation (1) occurs after a formal electron transfer to the nitrosyl ligand, achieved within an intense low-lying electron transition having a strong charge transfer character towards the strongly withdrawing nitrosyl, in any case.

Several research teams have targeted NO donors in the class of ruthenium-bipyridine ${ }^{12-14}$ and ruthenium-terpyridine ${ }^{12 a, 15-17}$ complexes, built up from ligands of different donating/accepting capabilities. As a part of this research effort, we have recently reported on various ruthenium-nitrosyl complexes based on the $[\mathrm{Ru}(\mathrm{tpy})(\mathrm{bpy})(\mathrm{NO})]^{3+} \operatorname{core}^{18}\left([\mathrm{RuT0B} 0]^{3+}\right.$ in Scheme 1$)$ initially investigated by Meyer et al. ${ }^{19}$ (tpy stands for 2,2':6',2''-terpyridine and bpy for 2,2'-bipyridine). These various attempts were motivated by the idea that introducing a donor (either fluorene, ${ }^{18 \mathrm{a}}$ carbazole, ${ }^{18 \mathrm{~b}}$ or methoxyphenyl ${ }^{18 c}$ ) on the terpyridine ligand would favor an intense charge transfer towards the RuNO fragment, thus increasing the quantum yield of NO release $\left(\phi_{\mathrm{NO}}=\right.$ number of $\mathrm{NO} \cdot$ generated $/$ number of photon absorbed by the complex). 
In the present contribution, we wish to target the role devoted to an electron donating substituent in the optical and photo-chemical properties of the ruthenium nitrosyl species through the investigation of a set of substitution achieved either on the terpyridine $\left([\mathrm{RuT} 1 \mathrm{~B} 0]^{3+}\right)$, on the bipyridine $\left([\mathrm{RuT} 0 \mathrm{~B} 2]^{3+}\right)$, and on both ligands $\left([\mathrm{RuT} 1 \mathrm{~B} 2]^{3+}\right)$, as shown in Scheme 1. After the report and whole characterization of the ruthenium complexes, their optical spectra will be presented experimentally and approached computationally, within the framework of the density functional theory (DFT) to determine the origin of the observed transitions. $\phi_{\mathrm{NO}}$ will be investigated by irradiation on the low-lying "push-pull" transitions, and tentatively analyzed in an attempt to provide new features towards a comprehensive description of the release process.

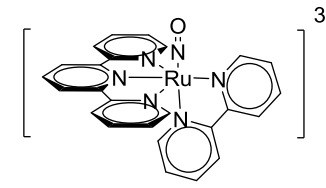

[RuTOB0] $^{3+}$

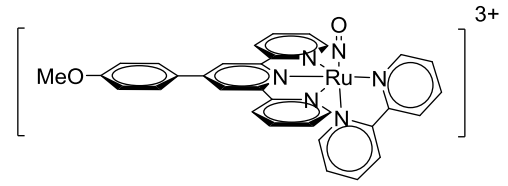

[RuT1B0] $^{3+}$
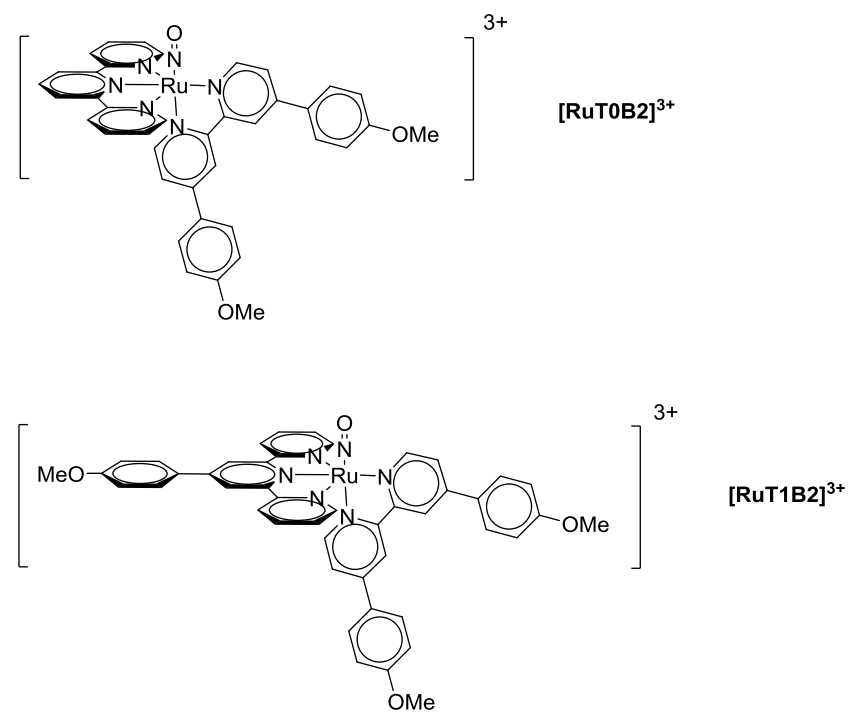

Scheme $1 \quad\left[\mathrm{Ru}(\text { terpy)(bipy)(NO) }]^{2+}\right.$ complexes with $0,1,2$, and 3 methoxyphenyl substituents.

\section{Experimental}

\section{Starting materials and equipment}

The 4,4'-bis(4-methoxyphenyl)-2,2'-bipyridine (MP 2 bipy ${ }^{20}$ and 4'-(4-methoxyphenyl)-2,2':6',2'”terpyridine (MPterpy) ${ }^{21}$ ligands were synthesized as previously described in the literature. Likewise, 
$\left[\mathrm{Ru}(\right.$ terpy $\left.)(\mathrm{Cl})_{3}\right]$ and $\left[\mathrm{Ru}(\mathrm{MPterpy})(\mathrm{Cl})_{3}\right]$ was synthetized following Adcock's procedure. ${ }^{22}$ Triethylamine (Sigma-Aldrich), $\mathrm{LiCl}$ (Alfa Aesar), Ethylene glycol (Fluka), $\mathrm{NH}_{4} \mathrm{PF}_{6}$ (Alfa Aesar), and $\mathrm{NaNO}_{2}$ (Fluka) were the highest purity grade and were used as received. The ${ }^{1} \mathrm{H}$ NMR and ${ }^{13} \mathrm{C}$ NMR spectra were recorded at $298 \mathrm{~K}$ with a Bruker Avance 400 spectrometer, using $\mathrm{CDCl}_{3}, \mathrm{CD}_{3} \mathrm{OD}$ or $\mathrm{CD}_{3} \mathrm{CN}$ as an internal reference. The IR spectra were recorded with a Perkin-Elmer (FTIR/FIR) 100 Spectrometer. The ESI mass spectra were performed on a UPLC Xevo G2 Q TOF (Waters) spectrometer. The UV-Vis spectra were obtained on a Perkin Elmer Lambda 35 UV-Vis spectrometer. For the deconvolution of the spectra, the shapes of the transitions were assumed to correspond to Gaussian functions while drawn against the transition energies. Elemental analyses were performed at LCC with a Perkin-Elmer 2400 series II Instrument. Electron paramagnetic resonance experiments (EPR) were performed on a Bruker ESP 500E spectrometer. The setting employed for the measurements was the same as that previously reported. ${ }^{18 \mathrm{c}}\left[\mathrm{Fe}(\mathrm{MGD})_{2}\right](\mathrm{MGD}=\mathrm{N}-$ methyl-Dglucamine dithiocarbamate) was used as a spin trapping reagent. ${ }^{23}$ The light source was a $250 \mathrm{~W}$ Oriel Hg lamp (Palaiseau, France).

\section{Synthesis}

The synthetic route towards [RuT0B2] $\left(\mathrm{PF}_{6}\right)_{3}$, and $[\mathrm{RuT0B} 2]\left(\mathrm{PF}_{6}\right)_{3}$ is summarized in Scheme 2. The atom labeling used for the ${ }^{1} \mathrm{H}$ - and $13 \mathrm{C}-\mathrm{NMR}$ assignments is provided as supplementary information (Figure S3).
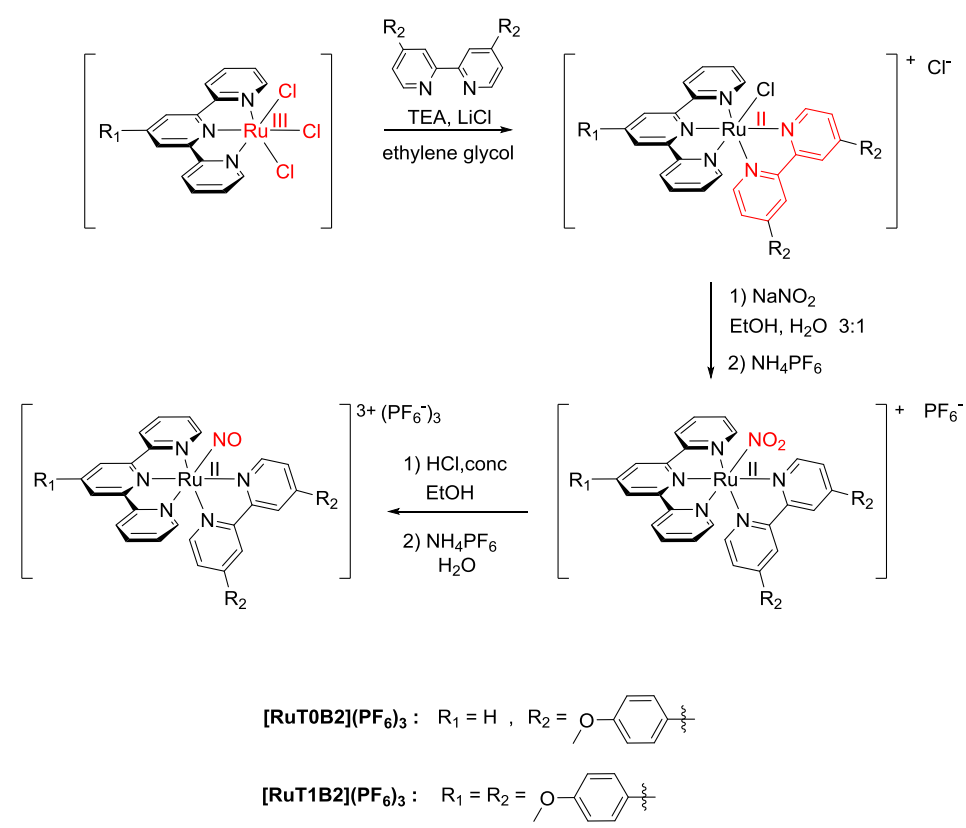

Scheme 2 Synthetic route towards $[\mathrm{RuT0B} 2]\left(\mathrm{PF}_{6}\right)_{3}$ and $[\mathrm{RuT0B} 2]\left(\mathrm{PF}_{6}\right)_{3}$. 
$\left[\mathrm{Ru}^{I I}(\right.$ terpy $)\left(\mathrm{MP}_{2}\right.$ bipy $\left.) C l\right](\mathrm{Cl})$. [Ru ${ }^{\mathrm{III}}($ terpy $\left.) \mathrm{Cl}_{3}\right](88 \mathrm{mg}, 0.2 \mathrm{mmol}), \mathrm{MP}_{2}$ bipy $(74 \mathrm{mg}, 0.2 \mathrm{mmol})$, lithium chloride (64 mg, $1.5 \mathrm{mmol})$, ethylene glycol $(27 \mathrm{~mL})$, and triethylamine $(0.24 \mathrm{~mL}, 1.7 \mathrm{mmol})$ were successively added into a $50 \mathrm{~mL}$ flask. The mixture was heated at $160^{\circ} \mathrm{C}$ during $4 \mathrm{~h}$. After a hot filtration, the mixture was evaporated to dryness under reduced pressure, at $120^{\circ} \mathrm{C}$. The residue obtained was washed with a large volume of water and sonicated, and finally filtered and dried under vacuum, providing $99 \mathrm{mg}(64 \%)$ of the desired compound, as a dark purple solid. ${ }^{1} \mathrm{H}$ NMR $\left(\mathrm{CD}_{3} \mathrm{OD}\right.$, $400 \mathrm{MHz}): \delta 10.15\left(1 \mathrm{H}, \mathrm{d}, \mathrm{H}_{6 \mathrm{~B}},{ }^{3} \mathrm{~J}_{6 / 5}=6.0 \mathrm{~Hz}\right) ; 9.17\left(1 \mathrm{H}, \mathrm{d}, \mathrm{H}_{3 \mathrm{~B}},{ }^{4} \mathrm{~J}_{3 / 5}=2.0 \mathrm{~Hz}\right) ; 8.88\left(1 \mathrm{H}, \mathrm{d}, \mathrm{H}_{3^{\prime} \mathrm{B}}\right.$, $\left.{ }^{4} \mathrm{~J}_{3^{\prime} / 5^{\prime}}=1.9 \mathrm{~Hz}\right) ; 8.66\left(2 \mathrm{H}, \mathrm{d}, \mathrm{H}_{3^{\prime} \mathrm{T}}+\mathrm{H}_{5^{\prime} \mathrm{T}},{ }^{3} \mathrm{~J}_{3^{\prime} / 4^{\prime}}={ }^{3} \mathrm{~J}_{5^{\prime} / 4^{\prime}}=8.1 \mathrm{~Hz}\right) ; 8.54\left(2 \mathrm{H}, \mathrm{d}, \mathrm{H}_{3 \mathrm{~T}}+\mathrm{H}_{3^{\prime},{ }^{\prime} \mathrm{T}},{ }^{3} \mathrm{~J}_{3 / 4}={ }^{3} \mathrm{~J}_{3^{\prime}, 4^{\prime}}\right.$, $=7.9 \mathrm{~Hz}) ; 8.28\left(1 \mathrm{H}, \mathrm{dd}, \mathrm{H}_{5 \mathrm{~B}},{ }^{3} \mathrm{~J}_{5 / 6}=6.1 \mathrm{~Hz},{ }^{4} \mathrm{~J}_{5 / 3}=1.9 \mathrm{~Hz}\right) ; 8.17-8.20\left(2 \mathrm{H}, \mathrm{m}, \mathrm{H}_{2 \mathrm{P}}+\mathrm{H}_{6 \mathrm{P}}\right) ; 8.14-8.17$

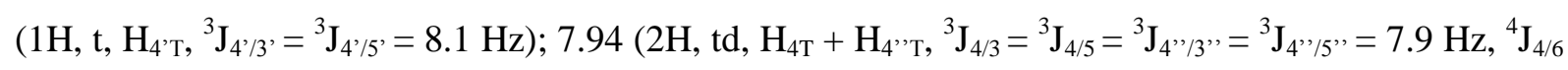

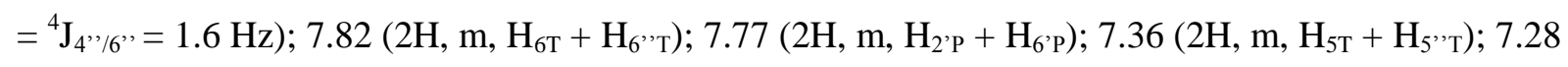
$\left(2 \mathrm{H}, \mathrm{m}, \mathrm{H}_{5}{ }^{\prime} \mathrm{B}+\mathrm{H}_{6^{\prime} \mathrm{B}}\right) ; 7.23\left(2 \mathrm{H}, \mathrm{m}, \mathrm{H}_{3 \mathrm{P}}+\mathrm{H}_{5 \mathrm{P}}\right) ; 7.02\left(2 \mathrm{H}, \mathrm{m}, \mathrm{H}_{3^{\prime} \mathrm{P}}+\mathrm{H}_{5}{ }^{\prime} \mathrm{P}\right) ; 3.95\left(3 \mathrm{H}, \mathrm{s}, \mathrm{OCH}_{3}\right) ; 3.82(3 \mathrm{H}$, $\left.\mathrm{s}, \mathrm{OCH}_{3}\right) .{ }^{13} \mathrm{C} \mathrm{NMR}\left(\mathrm{CD}_{3} \mathrm{OD}, 100 \mathrm{MHz}\right): \delta 163.18\left(\mathrm{C}_{4 \mathrm{P}}\right.$ or $\left.\mathrm{C}_{4}{ }^{\prime} \mathrm{P}\right) ; 163.05\left(\mathrm{C}_{4 \mathrm{P}}\right.$ or $\left.\mathrm{C}_{4}{ }^{\prime} \mathrm{P}\right) ; 160.61\left(\mathrm{C}_{2}{ }^{\prime} \mathrm{B}\right)$; $160.33\left(\mathrm{C}_{2 \mathrm{~T}}+\mathrm{C}_{2}{ }^{\prime}{ }_{\mathrm{T}}\right) ; 159.65\left(\mathrm{C}_{2}{ }^{\prime} \mathrm{T}+\mathrm{C}_{6}{ }^{\prime} \mathrm{T}\right) ; 158.10\left(\mathrm{C}_{2 \mathrm{~B}}\right) ; 153.47\left(\mathrm{C}_{6 \mathrm{~B}}\right) ; 153.36\left(\mathrm{C}_{6 \mathrm{~T}}+\mathrm{C}_{6}{ }{ }^{\prime} \mathrm{T}\right) ; 152.48$ $\left(\mathrm{C}_{6,}{ }^{\prime}\right) ; 149.94\left(\mathrm{C}_{4 \mathrm{~B}}\right) ; 149.01\left(\mathrm{C}_{4 \cdot{ }^{\prime} \mathrm{B}}\right) ; 138.30\left(\mathrm{C}_{4 \mathrm{~T}}+\mathrm{C}_{4}{ }{ }^{\prime} \mathrm{T}\right) ; 135.22\left(\mathrm{C}_{4}{ }^{\prime} \mathrm{T}\right) ; 130.04\left(\mathrm{C}_{2 \mathrm{P}}+\mathrm{C}_{6 \mathrm{P}}\right) ; 129.86$ $\left(\mathrm{C}_{1 \mathrm{P}}\right) ; 129.72\left(\mathrm{C}_{2}{ }^{\prime} \mathrm{P}+\mathrm{C}_{6}{ }^{\prime} \mathrm{P}\right) ; 129.13\left(\mathrm{C}_{1, \mathrm{P}}\right) ; 128.56\left(\mathrm{C}_{5 \mathrm{~T}}+\mathrm{C}_{5}{ }^{,}{ }_{\mathrm{T}}\right) ; 124.76\left(\mathrm{C}_{3 \mathrm{~T}}+\mathrm{C}_{3,{ }^{\prime} \mathrm{T}}\right) ; 124.69\left(\mathrm{C}_{5 \mathrm{~B}}\right) ;$ $124.08\left(\mathrm{C}_{5^{\prime} \mathrm{B}}\right) ; 123.65\left(\mathrm{C}_{3^{\prime} \mathrm{T}}+\mathrm{C}_{5}{ }^{\prime} \mathrm{T}\right) ; 121.48\left(\mathrm{C}_{3 \mathrm{~B}}\right) ; 121.35\left(\mathrm{C}_{3^{\prime} \mathrm{B}}\right) ; 115.97\left(\mathrm{C}_{3 \mathrm{P}}+\mathrm{C}_{5 \mathrm{P}}\right) ; 115.76\left(\mathrm{C}_{3^{\prime} \mathrm{P}}+\right.$ $\left.\mathrm{C}_{5}, \mathrm{P}\right) ; 55.34\left(\mathrm{OCH}_{3}\right) ; 55.24\left(\mathrm{OCH}_{3}\right)$. ESI-MS : m/z: $738.5[\mathrm{M}]^{+}$.

$\left[R u^{I I}(\right.$ terpy $)\left(M P_{2}\right.$ bipy $\left.)\left(N O_{2}\right)\right]\left(P F_{6}\right)$. [Ru ${ }^{\text {II }}$ terpy $)\left(\mathrm{MP}_{2}\right.$ bipy $\left.)(\mathrm{Cl})\right](\mathrm{Cl})(95 \mathrm{mg}, 0.12 \mathrm{mmol})$ was dissolved in a mixture of ethanol $(8.6 \mathrm{~mL})$ and water $(2.9 \mathrm{~mL})$. After complete dissolution, $\mathrm{NaNO}_{2}(83 \mathrm{mg}, 1.2$ mmol) was added and the violet resulting solution was heated under reflux for $3 \mathrm{~h} 30$. The solution turned dark red. After cooling down to room temperature, an excess of $\mathrm{NH}_{4} \mathrm{PF}_{6}(250 \mathrm{mg}, 1.53 \mathrm{mmol})$ dissolved in $1 \mathrm{~mL}$ of water was added. The solution was concentrated to three-quarters of its volume and the resulting solution was cooled in a fridge overnight. After a filtration and a washing with cold water, a dark red solid was dried under vacuum, affording $73 \mathrm{mg}(68 \%)$ of the desired compound. ${ }^{1} \mathrm{H}$ $\operatorname{NMR}\left(\mathrm{CD}_{3} \mathrm{CN}, 400 \mathrm{MHz}\right): \delta 9.82\left(1 \mathrm{H}, \mathrm{d}, \mathrm{H}_{6 \mathrm{~B}},{ }^{3} \mathrm{~J}_{6 / 5}=6.1 \mathrm{~Hz}\right) ; 8.93\left(1 \mathrm{H}, \mathrm{d}, \mathrm{H}_{3 \mathrm{~B}},{ }^{4} \mathrm{~J}_{3 / 5}=2.0 \mathrm{~Hz}\right) ; 8.69$ $\left(1 \mathrm{H}, \mathrm{d}, \mathrm{H}_{3^{\prime} \mathrm{B}},{ }^{4} \mathrm{~J}_{3^{\prime} / 5^{\prime}}=1.8 \mathrm{~Hz}\right) ; 8.49\left(2 \mathrm{H}, \mathrm{d}, \mathrm{H}_{3^{\prime} \mathrm{T}}+\mathrm{H}_{5^{\prime} \mathrm{T}},{ }^{3} \mathrm{~J}_{3^{\prime} / 4^{\prime}}={ }^{3} \mathrm{~J}_{5^{\prime} / 4^{\prime}}=8.0 \mathrm{~Hz}\right) ; 8.37\left(2 \mathrm{H}, \mathrm{d}, \mathrm{H}_{3 \mathrm{~T}}+\mathrm{H}_{3^{\prime}, \mathrm{T}}\right.$, $\left.{ }^{3} \mathrm{~J}_{3 / 4}={ }^{3} \mathrm{~J}_{3^{\prime}}, / 4^{\prime},=8.0 \mathrm{~Hz}\right) ; 8.21-8.16\left(2 \mathrm{H}, \mathrm{m}, \mathrm{H}_{5 \mathrm{~B}}+\mathrm{H}_{4^{\prime} \mathrm{T}}\right) ; 8.10\left(2 \mathrm{H}, \mathrm{m}, \mathrm{H}_{2 \mathrm{P}}+\mathrm{H}_{6 \mathrm{P}}\right) ; 7.94\left(2 \mathrm{H}, \mathrm{td}, \mathrm{H}_{4 \mathrm{~T}}+\right.$ $\left.\mathrm{H}_{4},{ }^{\prime},{ }^{3} \mathrm{~J}_{4 / 3}={ }^{3} \mathrm{~J}_{4 / 5}={ }^{3} \mathrm{~J}_{4}{ }^{\prime \prime} / 3^{\prime},={ }^{3} \mathrm{~J}_{4}, / 5,=7.8 \mathrm{~Hz},{ }^{4} \mathrm{~J}_{4 / 6}={ }^{4} \mathrm{~J}_{4}, / 6,=1.5 \mathrm{~Hz}\right) ; 7.83\left(2 \mathrm{H}, \mathrm{dd}, \mathrm{H}_{6 \mathrm{~T}}+\mathrm{H}_{6},{ }^{\prime} \mathrm{T},{ }^{3} \mathrm{~J}_{6 / 5}=\right.$

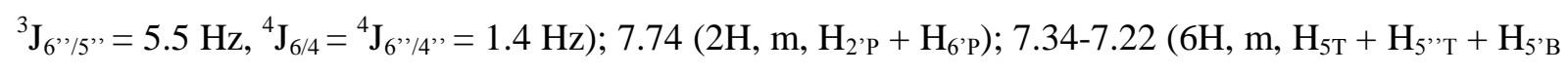
$\left.+\mathrm{H}_{6}{ }^{\circ} \mathrm{B}+\mathrm{H}_{3 \mathrm{P}}+\mathrm{H}_{5 \mathrm{P}}\right) ; 7.04\left(2 \mathrm{H}, \mathrm{m}, \mathrm{H}_{3}{ }^{\prime} \mathrm{P}+\mathrm{H}_{5}{ }^{\prime} \mathrm{P}\right) ; 3.94\left(3 \mathrm{H}, \mathrm{s}, \mathrm{OCH}_{3}\right) ; 3.83\left(3 \mathrm{H}, \mathrm{s}, \mathrm{OCH}_{3}\right)$

${ }^{13} \mathrm{C}$ NMR $\left(\mathrm{CD}_{3} \mathrm{CN}, 100 \mathrm{MHz}\right): \delta 162.69\left(\mathrm{C}_{4 \mathrm{P}}\right.$ ou $\left.\mathrm{C}_{4} \cdot \mathrm{P}\right) ; 162.62\left(\mathrm{C}_{4 \mathrm{P}}\right.$ ou $\left.\mathrm{C}_{4} \cdot \mathrm{P}\right) ; 159.56\left(\mathrm{C}_{2 \mathrm{~T}}+\mathrm{C}_{2}{ }^{,{ }^{\prime}}\right)$; $159.09\left(\mathrm{C}_{2}{ }^{\prime} \mathrm{T}+\mathrm{C}_{6}{ }^{\prime} \mathrm{T}\right) ; 158.14\left(\mathrm{C}_{2{ }^{\prime} \mathrm{B}}\right) ; 157.19\left(\mathrm{C}_{2 \mathrm{~B}}\right) ; 153.70\left(\mathrm{C}_{6 \mathrm{~B}}\right) ; 153.50\left(\mathrm{C}_{6 \mathrm{~T}}+\mathrm{C}_{6}{ }^{\prime}{ }^{\prime} \mathrm{T}\right) ; 151.81\left(\mathrm{C}_{6}{ }^{\prime} \mathrm{B}\right)$; $149.50\left(\mathrm{C}_{4}{ }^{\prime} \mathrm{B}\right) ; 149.20\left(\mathrm{C}_{4 \mathrm{~B}}\right) ; 138.74\left(\mathrm{C}_{4 \mathrm{~T}}+\mathrm{C}_{4}{ }^{\prime}{ }\right) ; 136.40\left(\mathrm{C}_{4}{ }^{\prime} \mathrm{T}\right) ; 129.91\left(\mathrm{C}_{2 \mathrm{P}}+\mathrm{C}_{6 \mathrm{P}}\right) ; 129.84\left(\mathrm{C}_{2}{ }^{\prime} \mathrm{P}+\right.$ $\left.\mathrm{C}_{6}{ }^{\prime} \mathrm{P}\right) ; 129.34\left(\mathrm{C}_{1 \mathrm{P}}\right) ; 128.72\left(\mathrm{C}_{1^{\prime} \mathrm{P}}\right) ; 128.44\left(\mathrm{C}_{5 \mathrm{~T}}+\mathrm{C}_{5}{ }^{\prime}{ }^{\prime} \mathrm{T}\right) ; 124.57\left(\mathrm{C}_{3 \mathrm{~T}}+\mathrm{C}_{3,{ }^{\prime} \mathrm{T}}+\mathrm{C}_{5 \mathrm{~B}}\right) ; 123.73\left(\mathrm{C}_{3, \mathrm{~T}}+\mathrm{C}_{5}{ }^{\prime} \mathrm{T}\right.$ $\left.+\mathrm{C}_{5}{ }^{\prime} \mathrm{B}\right) ; 121.57\left(\mathrm{C}_{3 \mathrm{~B}}\right) ; 121.10\left(\mathrm{C}_{3^{\prime} \mathrm{B}}\right) ; 115.92\left(\mathrm{C}_{3 \mathrm{P}}+\mathrm{C}_{5 \mathrm{P}}\right) ; 115.63\left(\mathrm{C}_{3^{\prime}, \mathrm{P}}+\mathrm{C}_{5^{\prime} \mathrm{P}}\right) ; 56.34\left(\mathrm{OCH}_{3}\right) ; 55.24$ 
$\left(\mathrm{OCH}_{3}\right)$. ESI-MS: m/z : $749.1[\mathrm{M}]^{+} ; 372\left[\mathrm{M}-\mathrm{NO}_{2}+\mathrm{CH}_{3} \mathrm{CN}\right]^{2+} ; 252 . \mathrm{IR}: v\left(\mathrm{~cm}^{-1}\right): 1387\left(v_{\mathrm{NO} 2}\right), 1346$ $\left(v_{\mathrm{NO} 2}\right), 839\left(v_{\mathrm{PF} 6}\right), 558\left(v_{\mathrm{PF} 6}\right)$.

[RuTOB2](PF $)_{3}$. $\left[\mathrm{Ru}(\right.$ terpy $\left.)\left(\mathrm{MP}_{2} \mathrm{bipy}\right)\left(\mathrm{NO}_{2}\right)\right]\left(\mathrm{PF}_{6}\right)(75 \mathrm{mg}, 0.083 \mathrm{mmol})$ was dissolved in a mixture of ethanol $(30 \mathrm{~mL})$ and $\mathrm{HCl} 37 \%(7 \mathrm{~mL})$. The resulting dark red-brown solution was heated at $60^{\circ} \mathrm{C}$ for 1 hour. The mixture was left at room temperature, and an excess of $\mathrm{NH}_{4} \mathrm{PF}_{6}(250 \mathrm{mg}, 1.53 \mathrm{mmol})$ dissolved in $3 \mathrm{~mL}$ of water was added. A beginning of precipitation was noticed. The solution was put in a fridge for $2 \mathrm{~h} 30$. Filtrating, washing with cold water, and eventually drying under vacuum afforded $75 \mathrm{mg}(77 \%)$ of a dark yellow solid. ${ }^{1} \mathrm{H}$ NMR $\left(\mathrm{CD}_{3} \mathrm{CN}, 400 \mathrm{MHz}\right): \delta 9.23\left(1 \mathrm{H}, \mathrm{d}, \mathrm{H}_{6 \mathrm{~B}},{ }^{3} \mathrm{~J}_{6 / 5}=6.2 \mathrm{~Hz}\right)$; $9.16\left(1 \mathrm{H}, \mathrm{d}, \mathrm{H}_{3 \mathrm{~B}},{ }^{4} \mathrm{~J}_{3 / 5}=2.0 \mathrm{~Hz}\right) ; 9.00\left(1 \mathrm{H}, \mathrm{m}, \mathrm{H}_{4}{ }^{\prime} \mathrm{T}\right) ; 8.95-8.92\left(3 \mathrm{H}, \mathrm{m}, \mathrm{H}_{3^{\prime} \mathrm{T}}+\mathrm{H}_{5^{\prime} \mathrm{T}}+\mathrm{H}_{3^{\prime} \mathrm{B}}\right) ; 8.76(2 \mathrm{H}, \mathrm{d}$, $\left.\mathrm{H}_{3 \mathrm{~T}}+\mathrm{H}_{3}{ }^{\prime}{ }^{\prime},{ }^{3} \mathrm{~J}_{3 / 4}={ }^{3} \mathrm{~J}_{3^{\prime}, / 4},=7.9 \mathrm{~Hz}\right) ; 8.51-8.45\left(3 \mathrm{H}, \mathrm{m}, \mathrm{H}_{4 \mathrm{~T}}+\mathrm{H}_{4}{ }^{\prime,} \mathrm{T}+\mathrm{H}_{5 \mathrm{~B}}\right) ; 8.24\left(2 \mathrm{H}, \mathrm{m}, \mathrm{H}_{2 \mathrm{P}}+\mathrm{H}_{6 \mathrm{P}}\right)$;

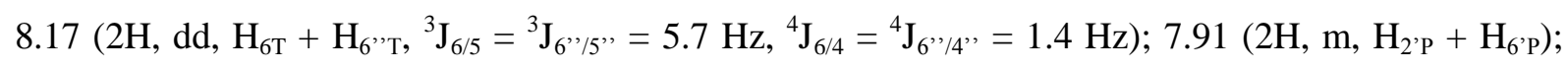
$7.76\left(2 \mathrm{H}, \mathrm{m}, \mathrm{H}_{5 \mathrm{~T}}+\mathrm{H}_{5^{\prime}, \mathrm{T}}\right) ; 7.62\left(1 \mathrm{H}, \mathrm{ddd}, \mathrm{H}_{5^{\prime} \mathrm{B}},{ }^{3} \mathrm{~J}_{5^{\prime} / 4^{\prime}}=7.8 \mathrm{~Hz},{ }^{3} \mathrm{~J}_{5^{\prime} / 6^{\prime}}=5.7 \mathrm{~Hz},{ }^{4} \mathrm{~J}_{5^{\prime} / \beta^{\prime}},=1.3 \mathrm{~Hz}\right) ; 7.31(2 \mathrm{H}$, $\left.\mathrm{m}, \mathrm{H}_{3 \mathrm{P}}+\mathrm{H}_{5 \mathrm{P}}\right) ; 7.13\left(2 \mathrm{H}, \mathrm{m}, \mathrm{H}_{3}{ }^{\prime} \mathrm{P}+\mathrm{H}_{5}{ }^{\prime} \mathrm{P}\right) ; 7.11\left(1 \mathrm{H}, \mathrm{m}, \mathrm{H}_{6}{ }^{\prime} \mathrm{B}\right) ; 4.00\left(3 \mathrm{H}, \mathrm{s}, \mathrm{OCH}_{3}\right) ; 3.97\left(3 \mathrm{H}, \mathrm{s}, \mathrm{OCH}_{3}\right)$. ${ }^{13} \mathrm{C}$ NMR $\left(\mathrm{CD}_{3} \mathrm{CN}, 100 \mathrm{MHz}\right): \delta 164.36\left(\mathrm{C}_{4}{ }^{\prime} \mathrm{P}\right) ; 164.20\left(\mathrm{C}_{4 \mathrm{P}}\right) ; 157.6\left(\mathrm{C}_{2 \mathrm{~T}}+\mathrm{C}_{2}{ }^{{ }{ }_{\mathrm{T}}}\right) ; 156.00\left(\mathrm{C}_{2}{ }^{\prime} \mathrm{B}\right)$; $155.66\left(\mathrm{C}_{6 \mathrm{~T}}+\mathrm{C}_{6}{ }^{\prime}{ }^{\prime} \mathrm{T}\right) ; 155.57\left(\mathrm{C}_{2 \mathrm{~B}}+\mathrm{C}_{4{ }^{\prime} \mathrm{B}}\right) ; 155.16\left(\mathrm{C}_{2}{ }^{\prime} \mathrm{T}+\mathrm{C}_{6}{ }^{\prime} \mathrm{T}\right) ; 154.69\left(\mathrm{C}_{4 \mathrm{~B}}\right) ; 153.44\left(\mathrm{C}_{6 \mathrm{~B}}\right) ; 148.31$

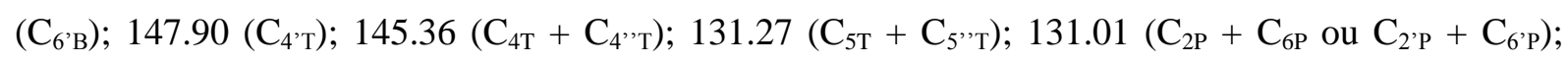
$130.95\left(\mathrm{C}_{2 \mathrm{P}}+\mathrm{C}_{6 \mathrm{P}}\right.$ ou $\left.\mathrm{C}_{2}{ }^{\prime} \mathrm{P}+\mathrm{C}_{6}{ }^{\prime} \mathrm{P}\right) ; 128.66\left(\mathrm{C}_{3 \mathrm{~T}}+\mathrm{C}_{3}{ }^{\prime}{ }_{\mathrm{T}}\right) ; 128.44\left(\mathrm{C}_{3^{\prime} \mathrm{T}}+\mathrm{C}_{5}{ }^{\prime} \mathrm{T}\right) ; 127.28\left(\mathrm{C}_{1 \mathrm{P}}\right) ; 127.18$ $\left(\mathrm{C}_{5 \mathrm{~B}}\right) ; 126.74\left(\mathrm{C}_{1}{ }^{\prime} \mathrm{P}\right) ; 126.24\left(\mathrm{C}_{5}{ }^{\prime} \mathrm{B}\right) ; 124.34\left(\mathrm{C}_{3 \mathrm{~B}}\right) ; 123.47\left(\mathrm{C}_{3^{\prime} \mathrm{B}}\right) ; 116.26\left(\mathrm{C}_{3 \mathrm{P}}+\mathrm{C}_{5 \mathrm{P}}\right) ; 116.14\left(\mathrm{C}_{3^{\prime} \mathrm{P}}+\right.$ $\left.\mathrm{C}_{5}, \mathrm{P}\right) ; 56.54\left(\mathrm{OCH}_{3}\right) ; 56.52\left(\mathrm{OCH}_{3}\right)$. IR: $v\left(\mathrm{~cm}^{-1}\right): 1937\left(v_{\mathrm{NO}}\right), 816\left(v_{\mathrm{PF} 6}\right), 552\left(v_{\mathrm{PF} 6}\right)$. ESI-MS: m/z: $360\left[\mathrm{Ru}(\text { terpy })\left(\mathrm{MP}_{2} \text { bpy }\right)\left(\mathrm{H}_{2} \mathrm{O}\right)\right]^{2+} ; \quad 372 \quad\left[\mathrm{Ru}(\text { terpy })\left(\mathrm{MP}_{2} \text { bpy }\right)\left(\mathrm{CH}_{3} \mathrm{CN}\right)\right]^{2+}$;

$\left[\mathrm{Ru}(\text { terpy })\left(\mathrm{MP}_{2} \text { bpy }\right)\left(\mathrm{NO}_{2}\right)\right]^{+}$. UV-Vis $\left(\mathrm{CH}_{3} \mathrm{CN}\right): \lambda_{\max }(\varepsilon): 229 \mathrm{~nm}\left(55000 \mathrm{~L} \mathrm{~mol}^{-1} \cdot \mathrm{cm}^{-1}\right), 292 \mathrm{~nm}(43000$ L.mol $\left.{ }^{-1} \cdot \mathrm{cm}^{-1}\right), 322 \mathrm{~nm}(\mathrm{sh}), 360 \mathrm{~nm}\left(33000 \mathrm{~L} \cdot \mathrm{mol}^{-1} \cdot \mathrm{cm}^{-1}\right.$ ). Anal. Calcd for $\mathrm{C}_{39} \mathrm{H}_{31} \mathrm{~F}_{18} \mathrm{~N}_{6} \mathrm{O}_{3} \mathrm{P}_{3} \mathrm{Ru}: \mathrm{C}$, 40.12; H, 2.68; N, 7.2. Found: C, 40.43; H, 2.34; N, 7.2.

[Ru(MPterpy)(MP $P_{2}$ bipy)Cl](Cl). [Ru(MPterpy)Cl $\left.{ }_{3}\right](137 \mathrm{mg}, 0.25 \mathrm{mmol}), \mathrm{MP}_{2}$ bipy (92 mg, 0.25 mmol), lithium chloride (80 mg, $1.875 \mathrm{mmol}$ [7.5 eq]), ethylene glycol (29 $\mathrm{mL})$, and triethylamine $(0.29 \mathrm{~mL}, 2.125 \mathrm{mmol})$ were successively introduced in a $50 \mathrm{~mL}$ flask. The mixture was heated at $170^{\circ} \mathrm{C}$ during $8 \mathrm{~h}$. After a hot filtration, the solution was evaporated to dryness under reduced pressure at $120^{\circ} \mathrm{C}$. The residue obtained was washed with a huge amount of water and sonicated. The resulting dark purple solid is filtered, washed with water, a minimum of ethanol and ether, and dried under vacuum, affording $180 \mathrm{mg}(82 \%)$ of the desired compound. ${ }^{1} \mathrm{H}$ NMR $\left(\mathrm{CD}_{3} \mathrm{OD}, 400 \mathrm{MHz}\right): \delta 10.20$ $\left(1 \mathrm{H}, \mathrm{d}, \mathrm{H}_{6 \mathrm{~B}},{ }^{3} \mathrm{~J}_{6 / 5}=6.0 \mathrm{~Hz}\right) ; 9.19\left(1 \mathrm{H}, \mathrm{d}, \mathrm{H}_{3 \mathrm{~B}},{ }^{4} \mathrm{~J}_{3 / 5}=1.9 \mathrm{~Hz}\right) ; 8.90\left(3 \mathrm{H}, \mathrm{m}, \mathrm{H}_{3^{\prime}}{ }_{\mathrm{T}}+\mathrm{H}_{5^{\prime} \mathrm{T}}+\mathrm{H}_{3^{\prime} \mathrm{B}} ; 8.69(2 \mathrm{H}\right.$,

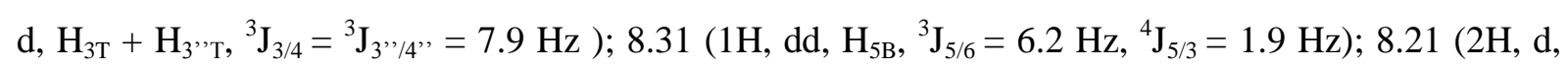
$\left.\mathrm{H}_{2 \mathrm{~Pb}}+\mathrm{H}_{6 \mathrm{~Pb}},{ }^{3} \mathrm{~J}_{2 / 3}={ }^{3} \mathrm{~J}_{6 / 5}=8.4 \mathrm{~Hz}\right) ; 8.15\left(2 \mathrm{H}, \mathrm{d}, \mathrm{H}_{2 \mathrm{Pt}}+\mathrm{H}_{6 \mathrm{Pt}},{ }^{3} \mathrm{~J}_{2 / 3}={ }^{3} \mathrm{~J}_{6 / 5}=8.3 \mathrm{~Hz}\right) ; 7.93\left(2 \mathrm{H}, \mathrm{m}, \mathrm{H}_{4 \mathrm{~T}}+\right.$ $\left.\mathrm{H}_{4},{ }^{\prime} \mathrm{T}\right) ; 7.84\left(2 \mathrm{H}, \mathrm{d}, \mathrm{H}_{6 \mathrm{~T}}+\mathrm{H}_{6^{\prime}, \mathrm{T}},{ }^{3} \mathrm{~J}_{6 / 5}={ }^{3} \mathrm{~J}_{6^{\prime}} / 5^{\prime},=5.4 \mathrm{~Hz}\right) ; 7.79\left(2 \mathrm{H}, \mathrm{d}, \mathrm{H}_{2^{\prime} \mathrm{Pb}}+\mathrm{H}_{6^{\prime} \mathrm{Pb}},{ }^{3} \mathrm{~J}_{2^{\prime} / 3^{\prime}},={ }^{3} \mathrm{~J}_{6^{\prime} / 5},=8.5\right.$ $\mathrm{Hz})$; 7.38-7.36 (3H, m, $\left.\mathrm{H}_{6{ }^{\prime} \mathrm{B}}+\mathrm{H}_{5 \mathrm{~T}}+\mathrm{H}_{5}{ }^{\prime}{ }^{\prime}\right)$; 7.31-7.29 (1H, m, $\left.\mathrm{H}_{5}{ }^{\prime} \mathrm{B}\right)$; 7.26-7.21 (4H, m, $\mathrm{H}_{3 \mathrm{~Pb}}+\mathrm{H}_{5 \mathrm{~Pb}}+$ 
$\left.\mathrm{H}_{3 \mathrm{Pt}}+\mathrm{H}_{5 \mathrm{Pt}}\right) ; 7.04\left(2 \mathrm{H}, \mathrm{m}, \mathrm{H}_{3^{\prime} \mathrm{Pb}}+\mathrm{H}_{5^{\prime} \mathrm{Pb}},{ }^{3} \mathrm{~J}_{3^{\prime} / 2^{\prime}}={ }^{3} \mathrm{~J}_{5^{\prime} / 6},=8.5 \mathrm{~Hz}\right) ; 3.97\left(3 \mathrm{H}, \mathrm{s}, \mathrm{OCH}_{3}\right) ; 3.96(3 \mathrm{H}, \mathrm{s}$, $\left.\mathrm{OCH}_{3}\right) ; 3.84\left(3 \mathrm{H}, \mathrm{s}, \mathrm{OCH}_{3}\right) .{ }^{13} \mathrm{C} \mathrm{NMR}\left(\mathrm{CD}_{3} \mathrm{OD}, 100 \mathrm{MHz}\right): \delta 161.79\left(\mathrm{C}_{4 \mathrm{~Pb}}\right) ; 161.65\left(\mathrm{C}_{4}{ }^{\mathrm{P} b}\right) ; 161.59$ $\left(\mathrm{C}_{4 \mathrm{Pt}}\right) ; 159.31\left(\mathrm{C}_{2}{ }^{\prime} \mathrm{B}\right) ; 159.18\left(\mathrm{C}_{2 \mathrm{~T}}+\mathrm{C}_{2}{ }{ }^{\prime} \mathrm{T}\right) ; 158.13\left(\mathrm{C}_{2}{ }^{\prime} \mathrm{T}+\mathrm{C}_{6}{ }^{\prime} \mathrm{T}\right) ; 156.82\left(\mathrm{C}_{2 \mathrm{~B}}\right) ; 152.12\left(\mathrm{C}_{6 \mathrm{~B}}\right) ; 152.00\left(\mathrm{C}_{6 \mathrm{~T}}\right.$ $\left.+\mathrm{C}_{6}{ }^{,{ }^{\prime}}\right) ; 151.09\left(\mathrm{C}_{6{ }^{\prime} \mathrm{B}}\right) ; 148.43\left(\mathrm{C}_{4 \mathrm{~B}}\right) ; 147.52\left(\mathrm{C}_{4{ }^{\prime} \mathrm{B}}\right) ; 146.55\left(\mathrm{C}_{4}{ }^{\prime} \mathrm{T}\right) ; 136.80\left(\mathrm{C}_{4 \mathrm{~T}}+\mathrm{C}_{4}{ }^{,{ }^{\prime}}\right) ; 128.95\left(\mathrm{C}_{1 \mathrm{Pt}}\right)$; $128.63\left(\mathrm{C}_{2 \mathrm{Pt}}+\mathrm{C}_{6 \mathrm{Pt}}+\mathrm{C}_{2 \mathrm{~Pb}}+\mathrm{C}_{6 \mathrm{~Pb}}\right) ; 128.50\left(\mathrm{C}_{1 \mathrm{~Pb}}\right) ; 128.30\left(\mathrm{C}_{2}{ }^{\prime} \mathrm{Pb}+\mathrm{C}_{6}{ }^{\prime} \mathrm{Pb}\right) ; 127.78\left(\mathrm{C}_{1}{ }^{\prime} \mathrm{Pb}\right) ; 127.04\left(\mathrm{C}_{5 \mathrm{~T}}+\right.$ $\left.\mathrm{C}_{5}{ }^{\prime}{ }^{\prime} \mathrm{T}\right) ; 123.54\left(\mathrm{C}_{3 \mathrm{~T}}+\mathrm{C}_{3}{ }^{\prime}{ }^{\prime} \mathrm{T}\right) ; 123.26\left(\mathrm{C}_{5 \mathrm{~B}}\right) ; 122.67\left(\mathrm{C}_{5^{\prime} \mathrm{B}}\right) ; 120.07\left(\mathrm{C}_{3 \mathrm{~B}}\right) ; 119.92\left(\mathrm{C}_{3^{\prime}{ }^{\prime} \mathrm{B}}\right) ; 119.29\left(\mathrm{C}_{3^{\prime} \mathrm{T}}+\right.$ $\left.\mathrm{C}_{5}, \mathrm{~T}\right) ; 114.57\left(\mathrm{C}_{3 \mathrm{~Pb}}+\mathrm{C}_{5 \mathrm{~Pb}}\right.$ or $\left.\mathrm{C}_{3 \mathrm{Pt}}+\mathrm{C}_{5 \mathrm{Pt}}\right) ; 114.55\left(\mathrm{C}_{3 \mathrm{~Pb}}+\mathrm{C}_{5 \mathrm{~Pb}}\right.$ or $\left.\mathrm{C}_{3 \mathrm{Pt}}+\mathrm{C}_{5 \mathrm{Pt}}\right) ; 114.34\left(\mathrm{C}_{3}{ }^{\prime} \mathrm{Pb}+\mathrm{C}_{5}{ }^{\prime} \mathrm{Pb}\right)$; $54.63\left(\mathrm{OCH}_{3} \times 2\right) ; 54.51\left(\mathrm{OCH}_{3}\right)$. ESI-MS : m/z : $844.4[\mathrm{M}]^{+}$.

$\left[R u(M P t e r p y)\left(M_{2}\right.\right.$ bipy $\left.)\left(N_{2}\right)\right]\left(P_{6}\right) . \quad\left[\mathrm{Ru}(\mathrm{MPterpy})\left(\mathrm{MP}_{2} \mathrm{bpy}\right)(\mathrm{Cl})\right] \mathrm{Cl}(130 \mathrm{mg}, 0.15 \mathrm{mmol})$ was dissolved in a mixture of ethanol $(12 \mathrm{~mL})$ and water $(4 \mathrm{~mL}) . \mathrm{NaNO}_{2}(96 \mathrm{mg}, 1.40 \mathrm{mmol})$ was added and the reaction mixture was heated under reflux during $4 \mathrm{~h} 20$. The dark brown solution was cooled down to room temperature, and an excess of $\mathrm{NH}_{4} \mathrm{PF}_{6}(350 \mathrm{mg}, 2.1 \mathrm{mmol})$ dissolved in $1 \mathrm{~mL}$ of water was added. The solution was concentrated to three-quarters of its volume, and the resulting solution was cooled in a fridge for $1 \mathrm{~h}$. After filtration and washing with cold water, the resulting dark brown solid was dried under vacuum in a desiccator. The crude compound (138 mg) was purified as following: the solid was dissolved in $135 \mathrm{~mL}$ of dichloromethane and the solution was filtrated. Petroleum ether $(550 \mathrm{~mL})$ was slowly added to the filtrate under stirring. The resulting mixture was put in a freezer for $3 \mathrm{~h}$. Filtrating, washing with cold petroleum ether and drying under vacuum provided $93 \mathrm{mg}(62 \%)$ of the desired compound, as a brown solid. ${ }^{1} \mathrm{H} \mathrm{NMR}\left(\mathrm{CD}_{3} \mathrm{CN}, 400 \mathrm{MHz}\right): \delta$ $9.87\left(1 \mathrm{H}, \mathrm{d}, \mathrm{H}_{6 \mathrm{~B}},{ }^{3} \mathrm{~J}_{6 / 5}=6.0 \mathrm{~Hz}\right) ; 8.97\left(1 \mathrm{H}, \mathrm{d}, \mathrm{H}_{3 \mathrm{~B}},{ }^{4} \mathrm{~J}_{3 / 5}=2.0 \mathrm{~Hz}\right) ; 8.73\left(1 \mathrm{H}, \mathrm{d}, \mathrm{H}_{3^{\prime} \mathrm{B}},{ }^{4} \mathrm{~J}_{3^{\prime} / 5},=2.0 \mathrm{~Hz}\right)$; $8.71\left(2 \mathrm{H}, \mathrm{s}, \mathrm{H}_{3}{ }^{\prime} \mathrm{T}+\mathrm{H}_{5}{ }^{\prime} \mathrm{T}\right) ; 8.50\left(2 \mathrm{H}, \mathrm{m}, \mathrm{H}_{3 \mathrm{~T}}+\mathrm{H}_{3}{ }{ }^{\prime} \mathrm{T}\right) ; 8.23\left(1 \mathrm{H}, \mathrm{dd}, \mathrm{H}_{5 \mathrm{~B}},{ }^{3} \mathrm{~J}_{5 / 6}=6.1 \mathrm{~Hz},{ }^{4} \mathrm{~J}_{5 / 3}=2.0 \mathrm{~Hz}\right)$; $8.14\left(2 \mathrm{H}, \mathrm{m}, \mathrm{H}_{2 \mathrm{~Pb}}+\mathrm{H}_{6 \mathrm{~Pb}}\right) ; 8.10\left(2 \mathrm{H}, \mathrm{m}, \mathrm{H}_{2 \mathrm{Pt}}+\mathrm{H}_{6 \mathrm{Pt}}\right) ; 7.96\left(2 \mathrm{H}, \mathrm{td}, \mathrm{H}_{4 \mathrm{~T}}+\mathrm{H}_{4},{ }^{\prime} \mathrm{T},{ }^{3} \mathrm{~J}_{4 / 3}={ }^{3} \mathrm{~J}_{4 / 5}={ }^{3} \mathrm{~J}_{4}{ }^{\prime \prime} / 3^{\prime},=\right.$ $\left.{ }^{3} \mathrm{~J}_{4}{ }^{\prime \prime} / 5^{\prime},=7.9 \mathrm{~Hz},{ }^{4} \mathrm{~J}_{4 / 6}={ }^{4} \mathrm{~J}_{4}{ }^{\prime \prime} / 6^{\prime},=1.6 \mathrm{~Hz}\right) ; 7.88\left(2 \mathrm{H}, \mathrm{m}, \mathrm{H}_{6 \mathrm{~T}}+\mathrm{H}_{6},{ }^{\prime}\right) ; 7.77\left(2 \mathrm{H}, \mathrm{m}, \mathrm{H}_{2}{ }^{\prime} \mathrm{Pb}+\mathrm{H}_{6}{ }^{\prime} \mathrm{Pb}\right) ; 7.39-$ $7.33\left(3 \mathrm{H}, \mathrm{m}, \mathrm{H}_{6,}{ }^{\prime} \mathrm{B}+\mathrm{H}_{5 \mathrm{~T}}+\mathrm{H}_{5}{ }^{\prime} \mathrm{T}\right) ; 7.30-7.23\left(5 \mathrm{H}, \mathrm{m}, \mathrm{H}_{5{ }^{\prime} \mathrm{B}}+\mathrm{H}_{3 \mathrm{~Pb}}+\mathrm{H}_{5 \mathrm{~Pb}}+\mathrm{H}_{3 \mathrm{Pt}}+\mathrm{H}_{5 \mathrm{Pt}}\right) ; 7.06(2 \mathrm{H}, \mathrm{m}$, $\left.\mathrm{H}_{3}{ }^{\prime} \mathrm{Pb}+\mathrm{H}_{5}{ }^{\prime} \mathrm{Pb}\right) ; 3.97\left(6 \mathrm{H}, \mathrm{s}, \mathrm{OCH}_{3} \times 2\right) ; 3.85\left(3 \mathrm{H}, \mathrm{s}, \mathrm{OCH}_{3}\right) \cdot{ }^{13} \mathrm{C} \mathrm{NMR}\left(\mathrm{CD}_{3} \mathrm{CN}, 100 \mathrm{MHz}\right): \delta 161.71$ $\left(\mathrm{C}_{4 \mathrm{~Pb}}\right.$ or $\mathrm{C}_{4}{ }^{\prime} \mathrm{Pb}$ or $\left.\mathrm{C}_{4 \mathrm{Pt}}\right) ; 161.61\left(\mathrm{C}_{4 \mathrm{~Pb}}\right.$ or $\mathrm{C}_{4}{ }^{\prime} \mathrm{Pb}$ or $\left.\mathrm{C}_{4 \mathrm{Pt}}\right) ; 161.60\left(\mathrm{C}_{4 \mathrm{~Pb}}\right.$ or $\mathrm{C}_{4}{ }^{\prime} \mathrm{Pb}$ or $\left.\mathrm{C}_{4 \mathrm{Pt}}\right) ; 158.79\left(\mathrm{C}_{2 \mathrm{~T}}+\mathrm{C}_{2}{ }^{,}{ }^{\mathrm{T}}\right)$; $157.91\left(\mathrm{C}_{2}{ }^{\prime} \mathrm{T}+\mathrm{C}_{6^{\prime} \mathrm{T}}\right) ; 157.23\left(\mathrm{C}_{2^{\prime} \mathrm{B}}\right) ; 156.28\left(\mathrm{C}_{2 \mathrm{~B}}\right) ; 152.70\left(\mathrm{C}_{6 \mathrm{~B}}\right) ; 152.58\left(\mathrm{C}_{6 \mathrm{~T}}+\mathrm{C}_{6^{\prime}{ }^{\prime} \mathrm{T}}\right) ; 150.74\left(\mathrm{C}_{6^{\prime} \mathrm{B}}\right)$; $148.47\left(\mathrm{C}_{4^{\prime} \mathrm{B}}\right) ; 148.11\left(\mathrm{C}_{4 \mathrm{~B}}\right) ; 147.43\left(\mathrm{C}_{4^{\prime} \mathrm{T}}\right) ; 137.63\left(\mathrm{C}_{4 \mathrm{~T}}+\mathrm{C}_{4}{ }^{\prime}{ }^{\prime} \mathrm{T}\right) ; 129.10\left(\mathrm{C}_{2 \mathrm{Pt}}+\mathrm{C}_{6 \mathrm{P}}\right) ; 129.00\left(\mathrm{C}_{1 \mathrm{Pt}}\right)$;

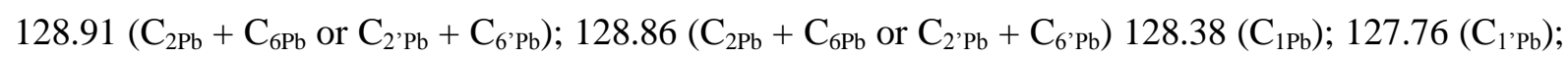
$127.35\left(\mathrm{C}_{5 \mathrm{~T}}+\mathrm{C}_{5}{ }^{\prime}{ }^{\prime}\right) ; 123.63\left(\mathrm{C}_{3 \mathrm{~T}}+\mathrm{C}_{3,{ }^{\prime}, \mathrm{T}}\right) ; 123.59\left(\mathrm{C}_{5 \mathrm{~B}}\right) ; 122.73\left(\mathrm{C}_{5^{\prime} \mathrm{B}}\right) ; 120.57\left(\mathrm{C}_{3 \mathrm{~B}}\right) ; 120.11\left(\mathrm{C}_{3^{\prime} \mathrm{B}}\right)$; $120.01\left(\mathrm{C}_{3}{ }^{\prime} \mathrm{T}+\mathrm{C}_{5}{ }^{\prime} \mathrm{T}\right) ; 114.93\left(\mathrm{C}_{3 \mathrm{~Pb}}+\mathrm{C}_{5 \mathrm{~Pb}}\right.$ or $\left.\mathrm{C}_{3 \mathrm{Pt}}+\mathrm{C}_{5 \mathrm{Pt}}\right) ; 114.86\left(\mathrm{C}_{3 \mathrm{~Pb}}+\mathrm{C}_{5 \mathrm{~Pb}}\right.$ or $\left.\mathrm{C}_{3 \mathrm{Pt}}+\mathrm{C}_{5 \mathrm{Pt}}\right) ; 114.63$ $\left(\mathrm{C}_{3^{\prime} \mathrm{Pb}}+\mathrm{C}_{5^{\prime} \mathrm{Pb}}\right) ; 55.35\left(\mathrm{OCH}_{3} \times 2\right) ; 55.24\left(\mathrm{OCH}_{3}\right) . \mathrm{IR}: v\left(\mathrm{~cm}^{-1}\right): 1334\left(v_{\mathrm{NO} 2}\right), 843\left(v_{\mathrm{PF} 6}\right), 826\left(v_{\mathrm{PF} 6}\right), 557$ $\left(v_{\mathrm{PF} 6}\right)$. ESI-MS : $\mathrm{m} / \mathrm{z}: 855.2[\mathrm{M}]^{+}$.

[RuT1B2] $\left(\mathrm{PF}_{6}\right)_{3}$. [Ru(MPterpy) $\left(\mathrm{MP}_{2}\right.$ bipy $\left.)\left(\mathrm{NO}_{2}\right)\right]\left(\mathrm{PF}_{6}\right)(60 \mathrm{mg}, 0.060 \mathrm{mmol})$ was dissolved in a mixture of ethanol $(22.5 \mathrm{~mL})$ and $\mathrm{HCl} 37 \%(5 \mathrm{~mL})$. The resulting brown solution was heated at $60^{\circ} \mathrm{C}$ 
for $1 \mathrm{~h}$. The solution was left to room temperature, and an excess of $\mathrm{NH}_{4} \mathrm{PF}_{6}(350 \mathrm{mg}, 2.13 \mathrm{mmol}$ ) dissolved in $3 \mathrm{~mL}$ of water was added. The resulting solution was evaporated under vacuum at a temperature maintained below $30^{\circ} \mathrm{C}$, until a beginning of precipitation occurred. The mixture was put in a fridge overnight. After filtration and washing with cold water, the resulting dark orange solid was dried under vaccum. (72 mg, $94 \%) .{ }^{1} \mathrm{H}$ NMR $\left(\mathrm{CD}_{3} \mathrm{CN}, 400 \mathrm{MHz}\right): \delta 9.23\left(1 \mathrm{H}, \mathrm{d}, \mathrm{H}_{6 \mathrm{~B}},{ }^{3} \mathrm{~J}_{6 / 5}=6.2 \mathrm{~Hz}\right)$; $9.13\left(1 \mathrm{H}, \mathrm{d}, \mathrm{H}_{3 \mathrm{~B}},{ }^{4} \mathrm{~J}_{3 / 5}=2.1 \mathrm{~Hz}\right) ; 9.08\left(2 \mathrm{H}, \mathrm{s}, \mathrm{H}_{3}{ }^{\prime} \mathrm{T}+\mathrm{H}_{5}{ }^{\prime} \mathrm{T}\right) ; 8.92-8.89\left(3 \mathrm{H}, \mathrm{m}, \mathrm{H}_{3{ }^{\prime} \mathrm{B}}+\mathrm{H}_{3 \mathrm{~T}}+\mathrm{H}_{3}{ }^{\prime}{ }^{\prime} \mathrm{T}\right) ; 8.51$ $\left(2 \mathrm{H}, \mathrm{td}, \mathrm{H}_{4 \mathrm{~T}}+\mathrm{H}_{4}{ }^{\prime \prime} \mathrm{T},{ }^{3} \mathrm{~J}_{4 / 3}={ }^{3} \mathrm{~J}_{4 / 5}={ }^{3} \mathrm{~J}_{4^{\prime \prime} / 3^{\prime}},={ }^{3} \mathrm{~J}_{4^{\prime \prime} / 5^{\prime}},=7.9 \mathrm{~Hz},{ }^{4} \mathrm{~J}_{4 / 6}={ }^{4} \mathrm{~J}_{4^{\prime \prime} / 6},=1.5 \mathrm{~Hz}\right) ; 8.47\left(1 \mathrm{H}, \mathrm{dd}, \mathrm{H}_{5 \mathrm{~B}}\right.$, $\left.{ }^{3} \mathrm{~J}_{5 / 6}=6.2 \mathrm{~Hz},{ }^{4} \mathrm{~J}_{5 / 3}=2.1 \mathrm{~Hz}\right) ; 8.32\left(2 \mathrm{H}, \mathrm{m}, \mathrm{H}_{2 \mathrm{Pt}}+\mathrm{H}_{6 \mathrm{Pt}}\right) ; 8.23\left(2 \mathrm{H}, \mathrm{m}, \mathrm{H}_{2 \mathrm{~Pb}}+\mathrm{H}_{6 \mathrm{~Pb}}\right) ; 8.17\left(1 \mathrm{H}, \mathrm{dd}, \mathrm{H}_{6 \mathrm{~T}}+\right.$ $\left.\mathrm{H}_{6^{\prime}, \mathrm{T}},{ }^{3} \mathrm{~J}_{6 / 5}={ }^{3} \mathbf{J}_{6^{\prime}, / 5},=5.6 \mathrm{~Hz},{ }^{4} \mathrm{~J}_{6 / 4}={ }^{4} \mathrm{~J}_{6^{\prime} / 4^{\prime},}=1.4 \mathrm{~Hz}\right) ; 7.90\left(2 \mathrm{H}, \mathrm{m}, \mathrm{H}_{2^{\prime} \mathrm{Pb}}+\mathrm{H}_{6}, \mathrm{~Pb}\right) ; 7.76\left(2 \mathrm{H}, \mathrm{m}, \mathrm{H}_{5 \mathrm{~T}}+\right.$ $\left.\mathrm{H}_{5}{ }^{\prime \prime} \mathrm{T}\right) ; 7.62\left(1 \mathrm{H}, \mathrm{dd}, \mathrm{H}_{5^{\prime} \mathrm{B}},{ }^{3} \mathrm{~J}_{5^{\prime} / 6}=6.4 \mathrm{~Hz},{ }^{4} \mathrm{~J}_{5^{\prime} / 3^{\prime}}=2.1 \mathrm{~Hz}\right) ; 7.36\left(2 \mathrm{H}, \mathrm{m}, \mathrm{H}_{3 \mathrm{Pt}}+\mathrm{H}_{5 \mathrm{Pt}}\right) ; 7.31\left(2 \mathrm{H}, \mathrm{m}, \mathrm{H}_{3 \mathrm{~Pb}}\right.$ $\left.+\mathrm{H}_{5 \mathrm{~Pb}}\right) ; 7.24\left(1 \mathrm{H}, \mathrm{d}, \mathrm{H}_{6^{\prime} \mathrm{B}},{ }^{3} \mathrm{~J}_{6^{\prime} / 5}, 6.4 \mathrm{~Hz}\right) ; 7.13\left(2 \mathrm{H}, \mathrm{m}, \mathrm{H}_{3^{\prime} \mathrm{Pb}}+\mathrm{H}_{5^{\prime} \mathrm{Pb}}\right) ; 4.02\left(3 \mathrm{H}, \mathrm{s}, \mathrm{OCH}_{3}\right) ; 4.00(3 \mathrm{H}, \mathrm{s}$, $\left.\mathrm{OCH}_{3}\right) ; 3.89\left(3 \mathrm{H}, \mathrm{s}, \mathrm{OCH}_{3}\right) .{ }^{13} \mathrm{C} \mathrm{NMR}\left(\mathrm{CD}_{3} \mathrm{CN}, 100 \mathrm{MHz}\right): \delta 163.90\left(\mathrm{C}_{4 \mathrm{Pt}}\right) ; 163.37\left(\mathrm{C}_{4}{ }^{\mathrm{P} P b}\right) ; 163.22$ $\left(\mathrm{C}_{4 \mathrm{~Pb}}\right) ; 158.07\left(\mathrm{C}_{4}{ }^{\prime} \mathrm{T}\right) ; 156.90\left(\mathrm{C}_{2 \mathrm{~T}}+\mathrm{C}_{2^{\prime}, \mathrm{T}}\right) ; 154.99\left(\mathrm{C}_{4}{ }^{\prime} \mathrm{B}\right) ; 154.63\left(\mathrm{C}_{6 \mathrm{~T}}+\mathrm{C}_{6}{ }^{\prime}{ }^{\prime} \mathrm{T}+\mathrm{C}_{2}{ }^{\prime} \mathrm{B}\right) ; 154.54\left(\mathrm{C}_{4 \mathrm{~B}}\right)$; $153.68\left(\mathrm{C}_{2}{ }^{\prime} \mathrm{T}+\mathrm{C}_{6}{ }^{\prime} \mathrm{T}\right) ; 153.61\left(\mathrm{C}_{2 \mathrm{~B}}\right) ; 152.33\left(\mathrm{C}_{6 \mathrm{~B}}\right) ; 147.34\left(\mathrm{C}_{6}{ }^{\prime} \mathrm{B}\right) ; 144.25\left(\mathrm{C}_{4 \mathrm{~T}}+\mathrm{C}_{4}{ }^{\prime}{ }_{\mathrm{T}}\right) ; 130.58\left(\mathrm{C}_{2 \mathrm{Pt}}+\right.$ $\left.\mathrm{C}_{6 \mathrm{Pt}}\right) ; 130.10\left(\mathrm{C}_{5 \mathrm{~T}}+\mathrm{C}_{5}{ }^{\prime}{ }_{\mathrm{T}}\right) ; 130.00\left(\mathrm{C}_{2 \mathrm{~Pb}}+\mathrm{C}_{6 \mathrm{~Pb}}\right.$ or $\left.\mathrm{C}_{2}{ }^{\prime} \mathrm{Pb}+\mathrm{C}_{6}{ }^{\prime} \mathrm{Pb}\right) ; 129.93\left(\mathrm{C}_{2 \mathrm{~Pb}}+\mathrm{C}_{6 \mathrm{~Pb}}\right.$ or $\left.\mathrm{C}_{2}{ }^{\prime} \mathrm{Pb}+\mathrm{C}_{6}{ }^{\prime} \mathrm{Pb}\right)$; $127.48\left(\mathrm{C}_{3 \mathrm{~T}}+\mathrm{C}_{3}{ }^{\prime}{ }_{\mathrm{T}}\right) ; 126.63\left(\mathrm{C}_{1 \mathrm{Pt}}\right) ; 126.30\left(\mathrm{C}_{1 \mathrm{~Pb}}\right) ; 126.21\left(\mathrm{C}_{5 \mathrm{~B}}\right) ; 125.76\left(\mathrm{C}_{1, \mathrm{~Pb}}\right) ; 125.25\left(\mathrm{C}_{5}{ }{ }\right) ; 123.53$ $\left(\mathrm{C}_{3^{\prime} \mathrm{T}}+\mathrm{C}_{5^{\prime} \mathrm{T}}\right) ; 123.28\left(\mathrm{C}_{3 \mathrm{~B}}\right) ; 122.42\left(\mathrm{C}_{3^{\prime} \mathrm{B}}\right) ; 115.54\left(\mathrm{C}_{3 \mathrm{Pt}}+\mathrm{C}_{5 \mathrm{Pt}}\right) ; 115.28\left(\mathrm{C}_{3 \mathrm{~Pb}}+\mathrm{C}_{5 \mathrm{~Pb}}\right) ; 115.14\left(\mathrm{C}_{3^{\prime} \mathrm{Pb}}+\right.$ $\left.\mathrm{C}_{5}{ }^{\mathrm{Pb}}\right) ; 55.73\left(\mathrm{OCH}_{3}\right) ; 55.58\left(\mathrm{OCH}_{3}\right) ; 55.52\left(\mathrm{OCH}_{3}\right)$. IR: $v\left(\mathrm{~cm}^{-1}\right): 1933\left(v_{\mathrm{NO}}\right), 829\left(v_{\mathrm{PF} 6}\right), 556\left(v_{\mathrm{PF} 6}\right)$. ESI-MS : m/z: $855.2\left[\mathrm{Ru}^{\mathrm{II}}(\mathrm{MPterpy})\left(\mathrm{MP}_{2} \text { bipy }\right)\left(\mathrm{NO}_{2}\right)\right]^{+} ; 425.1\left[\mathrm{Ru}^{\mathrm{II}}(\mathrm{MPterpy})\left(\mathrm{MP}_{2} \text { bipy }\right)\left(\mathrm{CH}_{3} \mathrm{CN}\right)\right]^{2+}$; $419.6 \quad\left[\mathrm{Ru}^{\mathrm{II}}(\mathrm{MPterpy})\left(\mathrm{MP}_{2} \text { bipy }\right)\left(\mathrm{NO}^{\circ}\right)\right]^{2+} ; \quad 413.6 \quad\left[\mathrm{Ru}^{\mathrm{II}}(\mathrm{MPterpy})\left(\mathrm{MP}_{2} \text { bipy }\right)\left(\mathrm{H}_{2} \mathrm{O}\right)\right]^{2+} ; \quad 404.6$ $\left[\mathrm{Ru}^{\mathrm{II}}(\mathrm{MPterpy})\left(\mathrm{MP}_{2} \text { bpy }\right)\right]^{2+} ; \quad 283.4 \quad\left[\mathrm{Ru}^{\mathrm{III}}(\mathrm{MPterpy})\left(\mathrm{MP}_{2} \mathrm{bpy}\right)\left(\mathrm{CH}_{3} \mathrm{CN}\right)\right]^{3+} ; \quad 269.4$ $\left[\mathrm{Ru}^{\text {III }}(\mathrm{MPterpy})\left(\mathrm{MP}_{2} \mathrm{bpy}\right)\right]^{3+}$. UV-Vis $\left(\mathrm{CH}_{3} \mathrm{CN}\right): \lambda_{\max }(\varepsilon): 227\left(52000 \mathrm{~L} \cdot \mathrm{mol}^{-1} . \mathrm{cm}^{-1}\right), 275$ (sh), 291 $\left(51000\right.$ L.mol $\left.{ }^{-1} \cdot \mathrm{cm}^{-1}\right), \quad 315 \quad(\mathrm{sh}), \quad 365 \quad\left(39000 \quad \mathrm{~L} \cdot \mathrm{mol}^{-1} \cdot \mathrm{cm}^{-1}\right), \quad 440 \quad(\mathrm{sh})$. Anal. Calcd for $\mathrm{C}_{46} \mathrm{H}_{37} \mathrm{~F}_{18} \mathrm{~N}_{6} \mathrm{O}_{4} \mathrm{P}_{3} \mathrm{Ru}: \mathrm{C}, 43.37 ; \mathrm{H}, 2.93 ; \mathrm{N}, 6.6$. Found: C, 43.25; H, 2.69; N, 6.47.

[Ru(terpy) $\left(\mathrm{MP}_{2}\right.$ bipy $\left.)\left(\mathrm{CH}_{3} \mathrm{CN}\right)\right]\left(\mathrm{PF}_{6}\right)_{2} .{ }^{1} \mathrm{H} \mathrm{NMR}\left(\mathrm{CD}_{3} \mathrm{CN}, 400 \mathrm{MHz}\right): \delta 9.55\left(1 \mathrm{H}, \mathrm{d}, \mathrm{H}_{6 \mathrm{~B}},{ }^{3} \mathrm{~J}_{6 / 5}=6.0\right.$ $\mathrm{Hz}) ; 8.97\left(1 \mathrm{H}, \mathrm{d}, \mathrm{H}_{3 \mathrm{~B}},{ }^{4} \mathrm{~J}_{3 / 5}=2.0 \mathrm{~Hz}\right) ; 8.70\left(1 \mathrm{H}, \mathrm{d}, \mathrm{H}_{3^{\prime} \mathrm{B}},{ }^{4} \mathrm{~J}_{3^{\prime} / 5^{\prime}}=2.1 \mathrm{~Hz}\right) ; 8.58\left(2 \mathrm{H}, \mathrm{d}, \mathrm{H}_{3^{\prime} \mathrm{T}}+\mathrm{H}_{5^{\prime} \mathrm{T}},{ }^{3} \mathrm{~J}_{3^{\prime} / 4^{\prime}}=\right.$ $\left.{ }^{3} \mathrm{~J}_{5^{\prime} / 4^{\prime}}=8.1 \mathrm{~Hz}\right) ; 8.44\left(2 \mathrm{H}, \mathrm{m}, \mathrm{H}_{3 \mathrm{~T}}+\mathrm{H}_{3^{\prime}, \mathrm{T}}\right) ; 8.32\left(1 \mathrm{H}, \mathrm{t}, \mathrm{H}_{4^{\prime} \mathrm{T}},{ }^{3} \mathrm{~J}_{4^{\prime} / 3^{\prime}}={ }^{3} \mathrm{~J}_{4^{\prime} / 5^{\prime}}=8.1 \mathrm{~Hz}\right) ; 8.19\left(1 \mathrm{H}, \mathrm{dd}, \mathrm{H}_{5 \mathrm{~B}}\right.$, $\left.{ }^{3} \mathrm{~J}_{5 / 6}=6.0 \mathrm{~Hz},{ }^{4} \mathrm{~J}_{5 / 3}=2.0 \mathrm{~Hz}\right) ; 8.13\left(2 \mathrm{H}, \mathrm{m}, \mathrm{H}_{2 \mathrm{Pbpy}}+\mathrm{H}_{6 \mathrm{Pbpy}}\right) ; 8.01\left(2 \mathrm{H}, \mathrm{td}, \mathrm{H}_{4 \mathrm{~T}}+\mathrm{H}_{4}{ }^{,{ }_{\mathrm{T}}},{ }^{3} \mathrm{~J}_{4 / 3}={ }^{3} \mathrm{~J}_{4 / 5}=\right.$ $\left.{ }^{3} \mathrm{~J}_{4}, / 3{ }^{\prime \prime}={ }^{3} \mathrm{~J}_{4}, / 5,=7.9 \mathrm{~Hz},{ }^{4} \mathrm{~J}_{4 / 6}={ }^{4} \mathrm{~J}_{4}, / 6 "=1.5 \mathrm{~Hz}\right) ; 7.81\left(2 \mathrm{H}, \mathrm{d}, \mathrm{H}_{6 \mathrm{~T}}+\mathrm{H}_{6},{ }^{\prime} \mathrm{T},{ }^{3} \mathrm{~J}_{6 / 5}={ }^{3} \mathrm{~J}_{6, "} / 5^{\prime \prime}=5.5\right.$

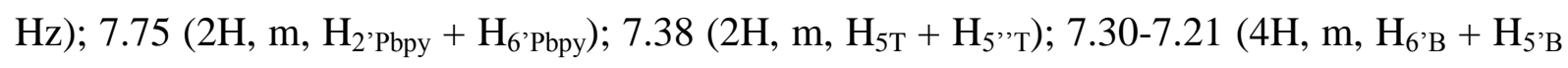
$\left.+\mathrm{H}_{3 \mathrm{Pbpy}}+\mathrm{H}_{5 \mathrm{Pbpy}}\right) ; 7.05\left(2 \mathrm{H}, \mathrm{m}, \mathrm{H}_{3}{ }^{\prime} \mathrm{Pbpy}+\mathrm{H}_{5}{ }^{\prime} \mathrm{Pbpy}\right) ; 3.95\left(3 \mathrm{H}, \mathrm{s}, \mathrm{OCH}_{3}\right) ; 3.83\left(3 \mathrm{H}, \mathrm{s}, \mathrm{OCH}_{3}\right)$.

$\left[R u(M P t e r p y)\left(M_{2}\right.\right.$ bipy) $\left.\left(C_{3} C N\right)\right]\left(P F_{6}\right)_{2} .{ }^{1} \mathrm{H} \mathrm{NMR}\left(\mathrm{CD}_{3} \mathrm{CN}, 400 \mathrm{MHz}\right): \delta 9.60\left(1 \mathrm{H}, \mathrm{d}, \mathrm{H}_{6 \mathrm{~B}},{ }^{3} \mathrm{~J}_{6 / 5}\right.$ $=6.0 \mathrm{~Hz}) ; 9.00\left(1 \mathrm{H}, \mathrm{m}, \mathrm{H}_{3 \mathrm{~B}},{ }^{4} \mathrm{~J}_{3 / 5}=2.1 \mathrm{~Hz}\right) ; 8.83\left(2 \mathrm{H}, \mathrm{s}, \mathrm{H}_{3}{ }^{\prime} \mathrm{T}+\mathrm{H}_{5}{ }^{\prime} \mathrm{T}\right) ; 8.74\left(1 \mathrm{H}, \mathrm{m}, \mathrm{H}_{3}{ }^{\prime} \mathrm{B}\right) ; 8.61$ $\left(2 \mathrm{H}, \mathrm{m}, \mathrm{H}_{3 \mathrm{~T}}+\mathrm{H}_{3}{ }^{\prime}{ }^{\mathrm{T}}\right) ; 8.23\left(1 \mathrm{H}, \mathrm{dd}, \mathrm{H}_{5 \mathrm{~B}},{ }^{3} \mathrm{~J}_{5 / 6}=6.0 \mathrm{~Hz},{ }^{4} \mathrm{~J}_{5 / 3}=2.0 \mathrm{~Hz}\right) ; 8.16\left(4 \mathrm{H}, \mathrm{m}, \mathrm{H}_{2 \mathrm{Pt}}+\mathrm{H}_{6 \mathrm{Pt}}\right.$ 


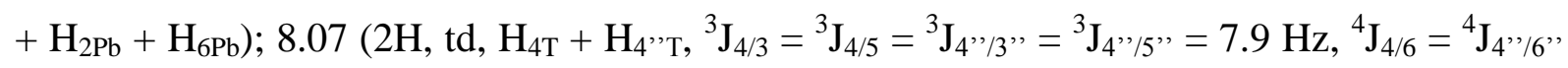

$=1.5 \mathrm{~Hz}) ; 7.85\left(2 \mathrm{H}, \mathrm{d}, \mathrm{H}_{6 \mathrm{~T}}+\mathrm{H}_{6}{ }^{\prime}{ }^{\prime},{ }^{3} \mathrm{~J}_{6 / 5}={ }^{3} \mathrm{~J}_{6}{ }^{\prime \prime} / 5,=5.5 \mathrm{~Hz}\right) ; 7.78\left(2 \mathrm{H}, \mathrm{m}, \mathrm{H}_{2}{ }^{\prime} \mathrm{Pb}+\mathrm{H}_{6}{ }^{\prime} \mathrm{Pb}\right) ; 7.40$

$\left(2 \mathrm{H}, \mathrm{m}, \mathrm{H}_{6}{ }^{\prime} \mathrm{B}+\mathrm{H}_{5}{ }^{\prime} \mathrm{B}\right) ; 7.34-7.27\left(6 \mathrm{H}, \mathrm{m}, \mathrm{H}_{5 \mathrm{~T}}+\mathrm{H}_{5}{ }^{\prime}{ }^{\prime} \mathrm{T}+\mathrm{H}_{3 \mathrm{Pt}}+\mathrm{H}_{5 \mathrm{Pt}}+\mathrm{H}_{3 \mathrm{~Pb}}+\mathrm{H}_{5 \mathrm{~Pb}}\right) ; 7.08(2 \mathrm{H}, \mathrm{m}$, $\left.\mathrm{H}_{3^{\prime} \mathrm{Pb}}+\mathrm{H}_{5}{ }^{\prime} \mathrm{Pb}\right) ; 3.99\left(3 \mathrm{H}, \mathrm{s}, \mathrm{OCH}_{3}\right) ; 3.98\left(3 \mathrm{H}, \mathrm{s}, \mathrm{OCH}_{3}\right) ; 3.86\left(3 \mathrm{H}, \mathrm{s}, \mathrm{OCH}_{3}\right)$.

\section{Computational details}

The four ruthenium-nitrosyl complexes were fully optimized using the Gaussian-09 program package $^{24}$ within the framework of the Density Functional Theory (DFT). In any case, the calculations were performed in the presence of acetonitrile, which was modelized by the Polarizable Continuum Model (SCRF=PCM method). ${ }^{25}$ The double- $\zeta$ basis set $6-31 G^{*}$ was used for all atoms except the heavy ruthenium atom, for which the LANL2DZ basis set was applied to account for relativistic effects. ${ }^{26}$ We have selected the hybrid functional B3PW91 for the optimization, for a better coherency with previous investigations. Indeed, B3PW91 has been shown to outperform other hybrid functionals (e.g. B3LYP) and pure functionals (e.g. PW91) in numerous cases of ruthenium complexes, ${ }^{27}$ especially when back bonding ligands (like NO) are present. ${ }^{28}$ In our previous investigation of $[\text { RuT1B0 }]^{3+}{ }^{18 c}$ several functionals were tested for the calculations of the UV-visible spectra (B3PW91, B3LYP, PBE0, and CAM-B3LYP) by time-dependent (TD)-DFT. CAM-B3LY ${ }^{29}$ was finally selected, for its better accuracy to reproduce the experimental transition energies $(<0.5 \mathrm{eV}$ in any case). Using the same conditions was found satisfactory here as well. The computed geometries of FT, cis- and trans-[Ru $\left.{ }^{\mathrm{II}}(\mathrm{FT}) \mathrm{Cl}_{2}(\mathrm{NO})\right]^{+}$are provided as Supplementary Information with the details of the related UV-visible computed spectra.

\section{Photochemical investigations}

Photochemistry: Kinetic studies on the photolysis reactions were carried out with a diode array Hewlett Packart 8452A spectrophotometer. Solutions of $2 \mathrm{~mL}$ of [RuT0B2] $\left(\mathrm{PF}_{6}\right)_{3}\left(2.33 \times 10^{-5} \mathrm{~mol} \mathrm{~L}^{-1}\right.$ for irradiation at $365 \mathrm{~nm})$ and $[\mathrm{RuT} 1 \mathrm{~B} 2]\left(\mathrm{PF}_{6}\right)_{3}\left(3.75 \times 10^{-5} \mathrm{~mol} \mathrm{~L}^{-1}\right.$ for irradiation at $\left.436 \mathrm{~nm}\right)$ in nondeoxygenated acetonitrile were used. The optical fiber was fixed laterally from the cuvette. Absorption spectra were taken after each 3 minutes, in fast scan mode, during a period of 21 hours, which allows reaching apparent stable absorption conditions. The UV-vis spectra were recorded under irradiation realized with a Muller reactor device equipped with a cooling water filter and a mercury arc lamp equipped with appropriate interference filter to isolate the desired irradiation wavelength $\left(\lambda_{\max }=365\right.$ $\mathrm{nm}$ or $436 \mathrm{~nm}$ ). The temperature was maintained at $25^{\circ} \mathrm{C}$ during the whole experiment. 
Quantum yield measurements: Light intensities were determined before each photolysis experiments by chemical actinometry procedure. The actinometers used were potassium ferrioxalate at $\lambda_{\text {irr }}=436 \mathrm{~nm}\left(\mathrm{I}_{0}=2.59 \times 10^{-7} \mathrm{~mol} \mathrm{~L}^{-1} \mathrm{~s}^{-1}\right)$, and at $\lambda_{\text {irr }}=365 \mathrm{~nm}\left(\mathrm{I}_{0}=5.30 \times 10^{-6} \mathrm{~mol} \mathrm{~L}^{-1} \mathrm{~s}^{-1}\right)$. The quantum yield $\left(\phi_{\mathrm{A}}\right)$ was determined by the program Sa3.3 written by D. Lavabre and V. Pimienta. ${ }^{30}$ It allows the resolution of the following differential equation:

$$
\frac{\mathrm{d}[\mathrm{A}]}{\mathrm{dt}}=-\Phi_{A} \mathrm{I}_{\mathrm{a}}^{\mathrm{A}}=-\Phi_{A} \operatorname{Abs}_{\mathrm{A}}^{\lambda} \mathrm{I}_{0} \mathrm{~F}
$$

where $I_{a}^{A}$ is the intensity of the light absorbed by the precursor, $\operatorname{Abs}_{\mathrm{A}}^{\lambda}$ the absorbance before irradiation, $\mathrm{Abs}_{\mathrm{Tot}}^{\lambda}$ the total absorbance, $\mathrm{I}_{0}$ the incident intensity measured at $436 \mathrm{~nm}$, and $\mathrm{F}$ the photokinetic factor given defined as:

$$
\mathrm{F}=\frac{\left(1-10^{-\mathrm{Abs}}{ }_{\mathrm{Tot}}^{\lambda}\right)}{\mathrm{Abs}_{\mathrm{Tot}}^{\lambda}}
$$

The equation (2) was fitted with the experimental data $\mathrm{Abs}_{\mathrm{Tot}}^{\lambda}=f(t)$ and 2 parameters $\phi_{\mathrm{A}}$ and $\varepsilon_{\mathrm{B}}\left(\varepsilon_{\mathrm{B}}\right.$ is the molar extinction coefficient measured at the end of the reaction $)$ at two wavelengths $\left(\lambda_{\text {irr }}=436\right.$ $\mathrm{nm}, \lambda_{\text {obs }}=470 \mathrm{~nm}$ for $[\mathrm{RuT1B} 2]\left(\mathrm{PF}_{6}\right)_{3} ; \lambda_{\text {irr }}=365 \mathrm{~nm}, \lambda_{\text {obs }}=420 \mathrm{~nm}$ for [RuT0B0] $\left.\left(\mathrm{PF}_{6}\right)_{3}\right)$ and $\lambda_{\text {irr }}=$ $365 \mathrm{~nm}, \lambda_{\text {obs }}=470 \mathrm{~nm}$ for $\left.[\mathrm{RuT0B} 2]\left(\mathrm{PF}_{6}\right)_{3}\right) . \lambda_{\text {obs }}$ was chosen because it corresponds to a large difference between molar extinction coefficient at the initial and final time of the photochemical reaction. Simulation and optimization procedures were performed by using numerical integration and a non-linear minimization algorithm for the fitting of the model to the experimental data ${ }^{30,31}$ It has to be pointed out that $\phi_{\mathrm{NO}}$ was previously published elsewhere for [RuT1B0] $\left(\mathrm{PF}_{6}\right)_{3}{ }^{18 \mathrm{c}}$ Nevertheless, we have found that this previous value was overestimated due to a wrong evaluation of $\mathrm{I}_{0}$. Contrary to the previous study, the $\mathrm{pH}$ of the solution was carefully lowered by addition of sulfuric acid in the actinometry procedure in the present case, thus leading to a better evaluation of $\mathrm{I}_{0}$ and a concomitant reduction of $\phi_{\mathrm{NO}}$. The experimental data reported here are all based on this new procedure.

Data for $[\mathrm{RuT0B0}]\left(\mathrm{PF}_{6}\right)_{3}$

$[\mathrm{A}]_{0}=8.6610^{-5} \mathrm{~mol} \mathrm{~L}^{-1}, \varepsilon_{A}^{365}=7500 \mathrm{~mol}^{-1} \mathrm{~L} \mathrm{~cm}^{-1}, \varepsilon_{A}^{420}=577 \mathrm{~mol}^{-1} \mathrm{~L} \mathrm{~cm}^{-1}$, $\varepsilon_{B}^{365}=2246 \mathrm{~mol}^{-1} \mathrm{~L} \mathrm{~cm}^{-1}, \varepsilon_{B}^{420}=6813 \mathrm{~mol}^{-1} \mathrm{~L} \mathrm{~cm}^{-1}$

Data for $[\mathrm{RuT} 0 \mathrm{~B} 2]\left(\mathrm{PF}_{6}\right)_{3}$

$[\mathrm{A}]_{0}=2.3310^{-5} \mathrm{~mol} \mathrm{~L}^{-1}, \varepsilon_{A}^{365}=31759 \mathrm{~mol}^{-1} \mathrm{~L} \mathrm{~cm}^{-1}, \varepsilon_{A}^{470}=3527 \mathrm{~mol}^{-1} \mathrm{~L} \mathrm{~cm}^{-1}$, $\varepsilon_{B}^{365}=14116 \mathrm{~mol}^{-1} \mathrm{~L} \mathrm{~cm}^{-1}, \varepsilon_{B}^{470}=18058 \mathrm{~mol}^{-1} \mathrm{~L} \mathrm{~cm}^{-1}$

Data for $[\mathrm{RuT} 1 \mathrm{~B} 2]\left(\mathrm{PF}_{6}\right)_{3}$

$[\mathrm{A}]_{0}=3.7610^{-5} \mathrm{~mol} \mathrm{~L}^{-1}, \varepsilon_{A}^{436}=17872 \mathrm{~mol}^{-1} \mathrm{~L} \mathrm{~cm}^{-1}, \varepsilon_{A}^{470}=8318 \mathrm{~mol}^{-1} \mathrm{~L} \mathrm{~cm}^{-1}$, 
$\varepsilon_{B}^{436}=15131 \mathrm{~mol}^{-1} \mathrm{~L} \mathrm{~cm}^{-1}, \varepsilon_{B}^{470}=20126 \mathrm{~mol}^{-1} \mathrm{~L} \mathrm{~cm}^{-1}$

\section{Electrochemical studies}

Electrochemical experiments were performed with a potentiostat Autolab PGSTAT100 (EcoChemie, The Netherlands) controlled by GPES 4.09 software. Experiments were carried out at room temperature in a homemade airtight three-electrode cell connected to a vacuum/argon line. The reference electrode was a saturated calomel electrode (SCE) separated from the solution by a bridge compartment. The counter electrode was a platinum wire of ca. $1 \mathrm{~cm}^{2}$ apparent surface. The working electrode was a Pt microdisk (radius $=0.25 \mathrm{~mm}$ ). The supporting electrolyte $(\mathrm{nBu} 4 \mathrm{~N})(\mathrm{PF} 6)$ (Fluka, 99\% electrochemical grade) was used as received and simply degassed under argon. Acetonitrile was freshly purified prior to use. The solutions used during the electrochemical studies were $10^{-3} \mathrm{~mol} \mathrm{~L}^{-1}$ and $10^{-1} \mathrm{~mol} \mathrm{~L}^{-1}$ respectively for the ruthenium complexes and for the supporting electrolyte. Before each measurement, the solutions were degassed by bubbling argon through them, and the working electrode was polished with a polishing machine (Presi P230). Typical instrumental parameters for recorded square wave voltammograms were: $\mathrm{SW}$ frequency $\mathrm{f}=20 \mathrm{~Hz}, \mathrm{SW}$ amplitude $\mathrm{Esw}=20 \mathrm{mV}$, and scan increment $\mathrm{dE}=5 \mathrm{mV}$. The irradiations performed prior recording the voltammograms of the photoproducts were performed with a mercury lamp. The voltammograms for the new [RuT0B2] $\left(\mathrm{PF}_{6}\right)_{3}$ and [RuT1B2] $\left(\mathrm{PF}_{6}\right)_{3}$ compounds and the complete redox potentials tables along the $[\mathrm{RuT0B} 0]\left(\mathrm{PF}_{6}\right)_{3}, \quad[\mathrm{RuT1B} 0]\left(\mathrm{PF}_{6}\right)_{3}, \quad[\mathrm{RuT0B} 2]\left(\mathrm{PF}_{6}\right)_{3}, \quad[\mathrm{RuT} 1 \mathrm{~B} 2]\left(\mathrm{PF}_{6}\right)_{3}$ series are provided as supplementary information.

\section{Results and discussion}

\section{Synthesis and characterization}

The synthesis of $[\mathrm{RuT0B} 2]\left(\mathrm{PF}_{6}\right)_{3}$ and $[\mathrm{RuT} 1 \mathrm{~B} 2]\left(\mathrm{PF}_{6}\right)_{3}$ reported here follows the general route previously reported for [RuT1B0] $\left(\mathrm{PF}_{6}\right)_{3}$ which implies the introduction of the ruthenium atom as $\mathrm{Ru}^{\mathrm{III}} \mathrm{Cl}_{3}$ followed by that of the nitrosyl ligand through a first reaction of a nitrite $\left(\mathrm{NO}_{2}{ }^{-}\right)$switched to $\mathrm{NO}^{+}$in acidic medium. ${ }^{18 \mathrm{c}}$ This synthetic procedure avoids the production of a large amount of undesirable homoleptic $\left[\mathrm{Ru}^{\mathrm{II}}(\text { terpy })_{2}\right]^{2+}$, species which was observed when $\mathrm{Ru}(\mathrm{NO})$ is introduced with the $\left[\mathrm{Ru}^{\mathrm{II}} \mathrm{Cl}_{5}(\mathrm{NO})\right]^{2-}$ reagent. ${ }^{32}$

Contrary to the synthesis of related complexes built up from unsubstituted bipyridines, ${ }^{18}$ the first step of the present synthesis (Scheme 2), requires the use of the rather insoluble $\mathrm{MP}_{2}$ bipy, which led to 
unexpected difficulties. Eventually, the reactions were successfully achieved in ethylene glycol, following a procedure previously used in our group in the case of ruthenium nitrosyl complexes with insoluble ligands. ${ }^{33}$ The use of an excess of triethylamine (TEA) is justified at this step to facilitate the reduction of $\mathrm{Ru}^{\mathrm{III}}$ to $\mathrm{Ru}^{\mathrm{II}}$ according to a mechanism previously suggested, ${ }^{34,35}$ which implies an electron transfer from TEA to the hexacoordinated ruthenium center without direct binding. Additionally, we have observed in most cases that a $\left[\mathrm{Ru}^{\mathrm{III}}\right.$ (terpy)(bipy)X] complex undergoes a spontaneous $\mathrm{Ru}^{\mathrm{III}} \rightarrow \mathrm{Ru}^{\mathrm{II}}$ reduction, even in the absence of clear reducing agent (vide infra). In the case of [RuT1B2] $\left(\mathrm{PF}_{6}\right)_{3}$, the substitution of $\mathrm{NO}_{2}^{-}$for $\mathrm{Cl}^{-}$required a purification step as previously reported by Snow et al..$^{22}$ The reaction steps 2 and 3 in Scheme 2 are easily monitored by ${ }^{1} \mathrm{H}-\mathrm{NMR}$ spectroscopy, from the observation of the shift of one proton located in position 6 on the bipyridine, spatially close to the monodentate ligands $\left(\mathrm{Cl}, \mathrm{NO}_{2}\right.$ or $\left.\mathrm{NO}\right)$, and therefore submitted to its deshielding influence. Thereby, switching from - $\mathrm{Cl}$ to $-\mathrm{NO}_{2}$ substituent and subsequently to -NO leads to the peakshifting of the proton in position 6 from $10.18 \mathrm{ppm}$ (in $\mathrm{CD}_{3} \mathrm{CN}$ ) to $9.82 \mathrm{ppm}$ (in $\mathrm{CD}_{3} \mathrm{CN}$ ) and finally to $9.23 \mathrm{ppm}$ (in $\mathrm{CD}_{3} \mathrm{CN}$ ) in the case of [RuT0B2] $\left(\mathrm{PF}_{6}\right)_{3}$ and from $10.20 \mathrm{ppm}$ (in $\mathrm{CD}_{3} \mathrm{OD}$ ) to $9.87 \mathrm{ppm}$ (in $\mathrm{CD}_{3} \mathrm{CN}$ ) and finally to $9.23 \mathrm{ppm}\left(\right.$ in $\mathrm{CD}_{3} \mathrm{CN}$ ) in the case of [RuT1B2] $\left(\mathrm{PF}_{6}\right)_{3}$.

Applying two-dimensional NMR-techniques HMBC, HMQC and COSY allows the complete assignment of the peaks observed in ${ }^{1} \mathrm{H}$ - and ${ }^{13} \mathrm{C}$ - NMR spectra for the six diamagnetic $\mathrm{Ru}(\mathrm{Cl})$, $\mathrm{Ru}\left(\mathrm{NO}_{2}\right)$, and $\mathrm{Ru}(\mathrm{NO})$-based species. The stretching vibrations ( $\left.v_{\mathrm{NO}}\right)$ are observed at $1933 \mathrm{~cm}^{-1}$ and $1937 \mathrm{~cm}^{-1}$ for [RuT1B2] $\left(\mathrm{PF}_{6}\right)_{3}$, and [RuT0B2] $\left(\mathrm{PF}_{6}\right)_{3}$, respectively. These values fall close to those of $1940 \mathrm{~cm}^{-1}$ and $1944 \mathrm{~cm}^{-1}$ previously recorded in $[\mathrm{RuT} 1 \mathrm{~B} 0]\left(\mathrm{PF}_{6}\right)_{3},{ }^{18 \mathrm{c}}$ and $[\mathrm{RuT0B} 0]\left(\mathrm{PF}_{6}\right)_{3} \cdot{ }^{33}$ Nevertheless, a tendency for decreasing frequencies is apparent when the number of methoxyphenyl donating substituent increases. This observation is in complete agreement with the idea that increasing the donating capabilities of the complexes gradually increases the charge transfer towards the antibonding $\pi^{*}$ level of the withdrawing nitrosyl, reduces its bond order and eventually its stretching frequency.

\section{Optical properties}

The UV-visible spectra recorded in acetonitrile are compared in Figure 1 for the four compounds under investigations. At first glance, a rough tendency for higher intensity is observed as the size of the $\pi$-skeleton of the ligands increases, from [RuT0B0] $\left(\mathrm{PF}_{6}\right)_{3}$ to $[\mathrm{RuT} 1 \mathrm{~B} 2]\left(\mathrm{PF}_{6}\right)_{3}$. More precisely, and apart from high energy transitions in the $250-300 \mathrm{~nm}$ range having likely $\pi \rightarrow \pi^{*}$ intra-ligand character, two main features are observed at about $375 \mathrm{~nm}$ and at about $425 \mathrm{~nm}$. The transition at $375 \mathrm{~nm}$ is present in the four complexes, with a tendency for higher intensity in the species containing the $\mathrm{MP}_{2}$ bipy ligand. The transition at $425 \mathrm{~nm}$ of weaker intensity is present on species containing the 
MPterpy ligand, only. Interestingly, the UV-visible spectrum of [RuT1B2] $\left(\mathrm{PF}_{6}\right)_{3}$ seems to result grossly from the sum of that of $[\mathrm{RuT1B} 0]\left(\mathrm{PF}_{6}\right)_{3}$, and that of $[\mathrm{RuT0B} 2]\left(\mathrm{PF}_{6}\right)_{3}$. This observation suggests that, to a large extent, the origin of the electronic transition at $425 \mathrm{~nm}$ is roughly the same for both [RuT1B2] $\left(\mathrm{PF}_{6}\right)_{3}$, and [RuT0B2] $\left(\mathrm{PF}_{6}\right)_{3}$. This idea is farther developed by a DFT analysis.

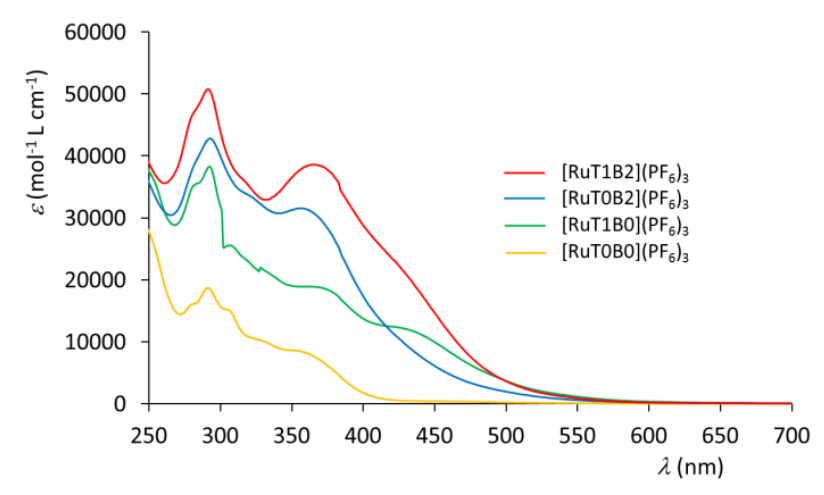

Figure 1 Comparison of the UV-visible spectra of the ruthenium complexes, in acetonitrile.

\section{DFT analyses}

Before discussing the computed optical properties, it is interesting to comment the evolution of few relevant data from the ground state optimizations in order to evaluate the charge transfer features along the $[\mathrm{Ru}(\mathrm{NO})]$ series. They are gathered in Table 1.

Table 1 Relevant data in the DFT-computed ruthenium nitrosyl complexes in the ground state: NO bond length ( $d_{\mathrm{NO}}$ in $\AA$ ), NO stretching frequency $\left(v_{\mathrm{NO}}\right.$ in $\left.\mathrm{cm}^{-1}\right)$, and torsion angle between the methoxyphenyl and the related pyridine rings $\left(\alpha\right.$ in $\left.{ }^{\circ}\right)$.

\begin{tabular}{|c|c|c|c|c|c|}
\hline Compound & $\mathrm{MP}$ & $d_{\mathrm{NO}}$ & $v_{\mathrm{NO}}$ & c & \\
\hline & & & & MP-terpy & $\mathrm{MP}_{2}$-bipy \\
\hline$[\mathrm{RuT0B} 0]^{3+}$ & 0 & 1.132 & 2054 & & \\
\hline$[\mathrm{RuT1B} 0]^{3+}$ & 1 & 1.134 & 2043 & 28.81 & \\
\hline$[\mathrm{RuT0B} 2]^{3+}$ & 2 & 1.133 & 2045 & & $26.70(\mathrm{t})^{\mathrm{a}}$ and $28.28(\mathrm{c})^{\mathrm{b}}$ \\
\hline$[\mathrm{RuT1B} 2]^{3+}$ & 3 & 1.135 & 2033 & 29.13 & $27.09(\mathrm{t})^{\mathrm{a}}$ and $28.57(\mathrm{c})^{\mathrm{b}}$ \\
\hline
\end{tabular}

\footnotetext{
${ }^{a}$ methoxyphenyl on the pyridine in trans position with respect to the $\mathrm{Ru}(\mathrm{NO})$.

${ }^{\mathrm{b}}$ methoxyphenyl on the pyridine in cis position with respect to the $\mathrm{Ru}(\mathrm{NO})$.
} 
As previously discussed, the charge transfer effects on $\mathrm{NO}$ (increasing $d_{\mathrm{NO}}$, and decreasing $v_{\mathrm{NO}}$ values) are more pronounced when the number of MP donor units increases. Nevertheless, the opposite is observed on the donating counterparts. Indeed, the MP to pyridine charge transfer is reduced (increasing torsion angle) when the number of donors increases from 0 to 3 . This evolution is readily understood from the fact that the withdrawing capability of a single NO is distributed on several donors, thus leading to a reduction of the effect on each individual donor. Additionally, the data gathered in Table 2 suggest that the effect of one substitution achieved on the terpyridine ligand is as efficient as that of two substitutions achieved on the bipyridine. A similar behavior has previously been observed in our group on a fluorenyl-based $\mathrm{Ru}(\mathrm{NO})$ complex. ${ }^{33}$

The TD-DFT computed spectra are compared in Figure 2, for the four $[\mathrm{RuT0B} 0]^{3+}$, [RuT1B0] $]^{3+}$, $[\text { RuT0B2 }]^{3+}$, and $[\text { RuT1B2 }]^{3+}$ complexes. Although some discrepancies are apparent in the general course between the theoretical and experimental spectra, it is possible to point out few computational features supporting the experimental data: (i) the tendency for higher overall intensity as the size of the $\pi$-skeleton of the ligands increases is evidenced in full agreement with the experiment; (ii) the presence of a sizeable low-lying transition at $\lambda>400 \mathrm{~nm}$, is observed in the only complexes in which a donor is present on the terpyridine (MPterpy); and (iii) the spectrum of [RuT1B2 $]^{3+}$ seems to result from the sum of those of $[\mathrm{RuT1B} 0]^{3+}$ and $[\mathrm{RuT0B} 2]^{3+}$, in the low-lying $375-425 \mathrm{~nm}$ wavelength domain. More precisely, the transitions at $413 \mathrm{~nm}$ and $382 \mathrm{~nm}$ computed in [RuT1B2] ${ }^{3+}$ seem to correspond to those computed at $417 \mathrm{~nm}$ and $394 \mathrm{~nm}$ in $[\mathrm{RuT1B} 0]^{3+}$ and $[\mathrm{RuT0B} 2]^{3+}$, respectively. The detailed analysis of these transitions is provided in Table 2 , to check out this hypothesis.

The data gathered in Table 2 lead to the suggestion that the two dominant transitions of [RuT1B2 $]^{3+}(1$ $\rightarrow 5$ and $1 \rightarrow 7)$ are reminiscent of those of $[\operatorname{RuT1B} 0]^{3+}(1 \rightarrow 3)$ and $[\operatorname{RuT0B} 2]^{3+}(1 \rightarrow 5)$, respectively. Indeed, they are found to possess roughly the same energies, relative intensities and charge transfer properties. The comparison of these transitions is farther depicted at the molecular level in Figure 3. It allows concluding unambiguously that the two transitions present in [RuT1B2 $]^{3+}$ are those of the parent $[\mathrm{RuT} 1 \mathrm{~B} 0]^{3+}$ and $[\mathrm{RuT0B} 2]^{3+}$ species without any significant mixing. This absence of mixing can be tentatively related to the symmetries of the orbitals involved in the transitions. 

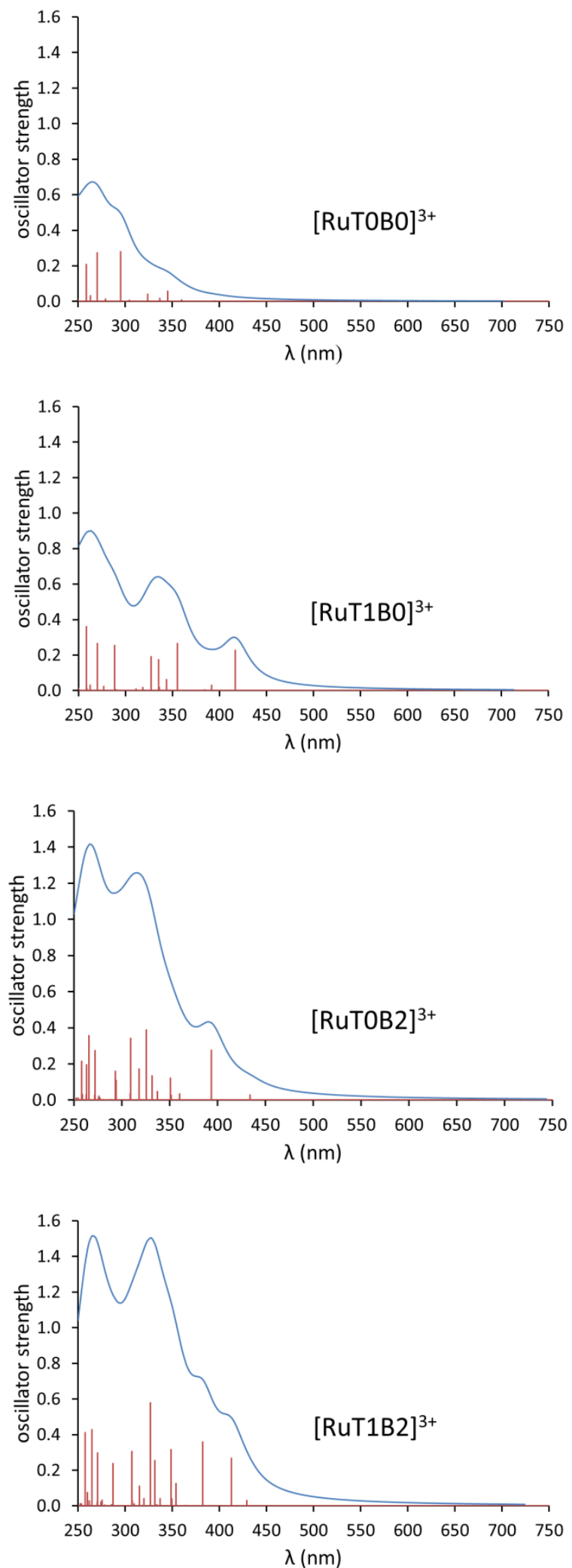

Figure 2 Comparison of the UV-visible spectra computed at the CAM-B3LYP/6-31G* level for the ruthenium nitrosyl complexes. 
Table 2 Details for the low energy transitions involved in the TD-DFT computed spectra of the ruthenium-nitrosyl complexes: absorption maxima $\left(\lambda_{\max }\right)$, oscillator strengths $(f)$, composition of the configuration interactions $(\mathrm{CI})$, and characters.

\begin{tabular}{|c|c|c|c|c|c|}
\hline Compound & Transition & $\lambda_{\max }(\mathrm{nm})$ & $\mathrm{f}$ & CI expansion ${ }^{a}$ & main character \\
\hline [RuT0B0] $^{3+}$ & $\begin{array}{l}1 \rightarrow 7 \\
1 \rightarrow 10\end{array}$ & $\begin{array}{l}345 \\
324\end{array}$ & $\begin{array}{l}0.058 \\
0.042\end{array}$ & $\begin{array}{l}59 \% \chi_{116 \rightarrow 117}+14 \% \chi_{112 \rightarrow 117} \\
88 \% \chi_{115 \rightarrow 118}\end{array}$ & $\begin{array}{l}\text { terpy } \rightarrow \mathrm{Ru}(\mathrm{NO}) \\
\text { bipy } \rightarrow \mathrm{Ru}(\mathrm{NO})\end{array}$ \\
\hline$\left[\right.$ RuT1B0] ${ }^{3+}$ & $1 \rightarrow 3$ & 417 & 0.227 & $76 \% \chi_{144 \rightarrow 145}$ & $\mathrm{MP}_{(\text {terpy })} \rightarrow \mathrm{Ru}(\mathrm{NO})$ \\
\hline [RuT0B2] $^{3+}$ & $\begin{array}{l}1 \rightarrow 3 \\
1 \rightarrow 5\end{array}$ & $\begin{array}{l}434 \\
393\end{array}$ & $\begin{array}{l}0.029 \\
0.276\end{array}$ & $\begin{array}{l}33 \% \chi_{172 \rightarrow 174}+32 \% \chi_{166 \rightarrow 174} \\
47 \% \chi_{171 \rightarrow 174}+31 \% \chi_{172 \rightarrow 174}\end{array}$ & $\begin{array}{l}\mathrm{MP}_{(\text {bipy })}+\text { bipy } \rightarrow \mathrm{Ru}(\mathrm{NO}) \\
\mathrm{MP}_{(\text {bipy })} \rightarrow \mathrm{Ru}(\mathrm{NO})\end{array}$ \\
\hline [RuT1B2] $^{3+}$ & $\begin{array}{l}1 \rightarrow 5 \\
1 \rightarrow 7\end{array}$ & $\begin{array}{l}413 \\
382\end{array}$ & $\begin{array}{l}0.269 \\
0.359\end{array}$ & $\begin{array}{l}65 \% \chi_{198 \rightarrow 201} \\
37 \% \chi_{199 \rightarrow 202}+36 \% \chi_{200 \rightarrow 202}\end{array}$ & $\begin{array}{l}\mathrm{MP}_{\text {(terpy) }} \rightarrow \mathrm{Ru}(\mathrm{NO}) \\
\mathrm{MP}_{(\text {bipy })} \rightarrow \mathrm{Ru}(\mathrm{NO})\end{array}$ \\
\hline
\end{tabular}

a orbital 116(117), 144(145), 172(173), and 200(201) are the HOMO(LUMO) in $[\text { RuT0B0 }]^{3+},[\text { RuT1B0 }]^{3+}$, [RuT0B2 $]^{3+}$, and $[\text { RuT1B2 }]^{3+}$, respectively. 


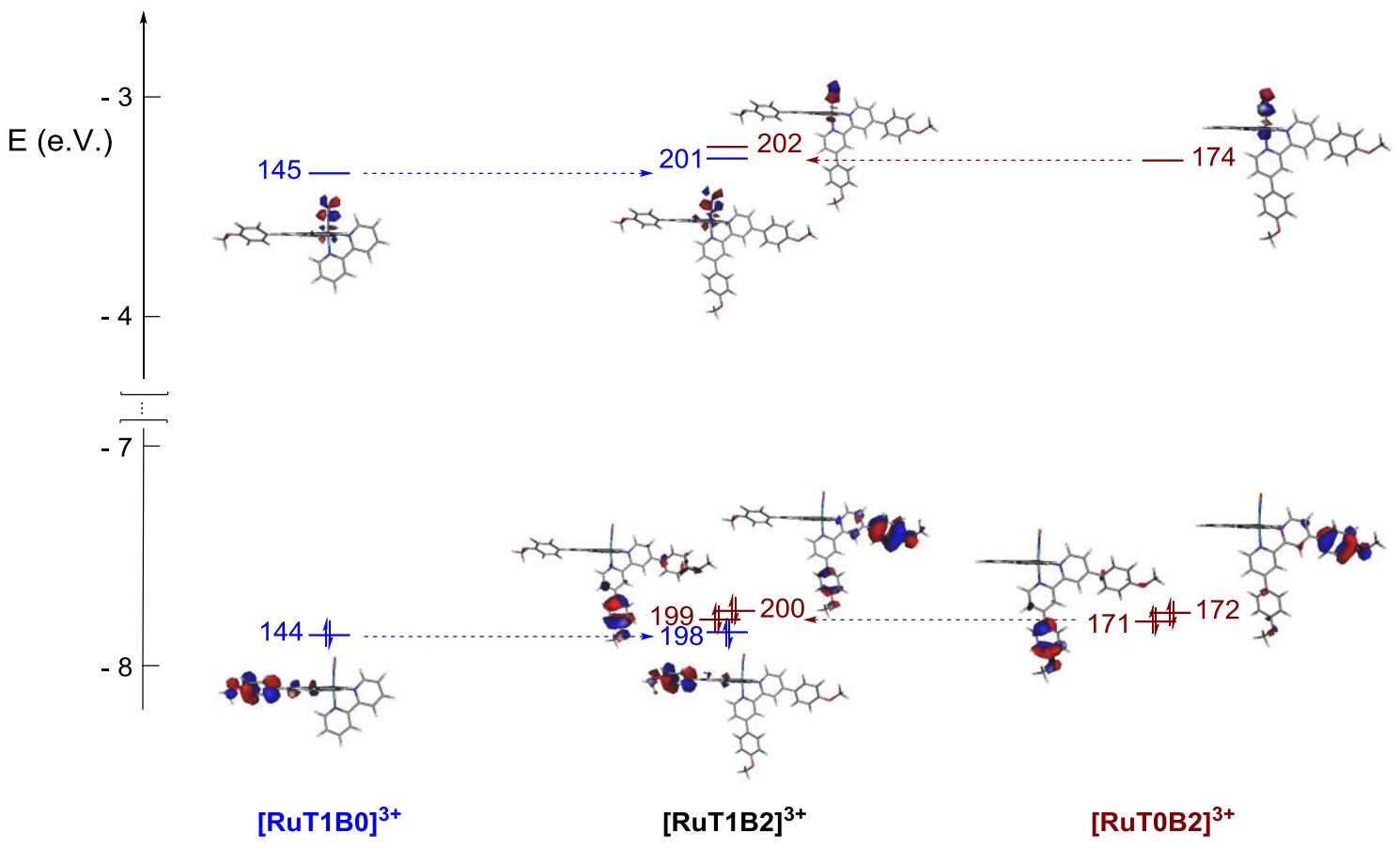

Figure 3 Main orbitals involved in the dominant low-energy transitions of [RuT1B0] ${ }^{3+}$, $[\mathrm{RuT0B} 2]^{3+}$, and [RuT1B2] $]^{3+}$.

Within the assumption of an ideal octahedral $\mathrm{RuN}_{6}$ coordination sphere, schematized in Scheme 3, the terpyridine ligand appears to lie in the $\mathrm{XZ}$ plane and the bipyridine ligand to lie in the $\mathrm{XY}$ plane. In $[\mathrm{RuT1B} 0]^{3+}$, the dominant $1 \rightarrow 3$ transition possesses a $\pi_{\mathrm{Y}}$ (terpy) to $d_{\mathrm{XY}} \pi_{\mathrm{X}}{ }^{*}$ character, while the dominant $1 \rightarrow 5$ transition of $[\mathrm{RuT0B} 2]^{3+}$ possesses a $\pi_{\mathrm{Z} \text { (bipy) }}$ to $d_{\mathrm{YZ}} \pi_{\mathrm{Z}}{ }^{*}$ character. Both charge transfer phenomena being strictly orthogonal, it is therefore not surprising that they found their counterpart in the $[\mathrm{RuT} 1 \mathrm{~B} 2]^{3+}$, without any mixing.

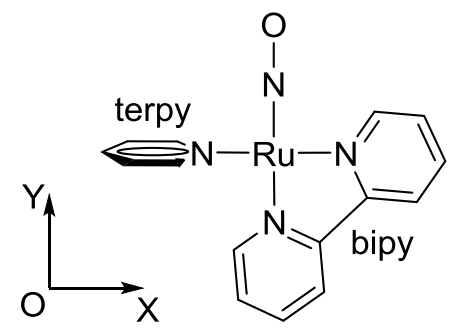

Scheme 3 Partial coordination sphere in the XY plane for a $\left[\mathrm{Ru}(\text { terpy)(bipy)(NO) }]^{3+}\right.$ species in the case of an ideal $\mathrm{RuN}_{6}$ octahedral symmetry. 


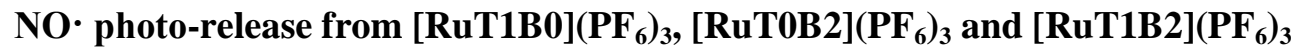

Numerous ruthenium nitrosyl complexes have been observed to release NO under irradiation, with very different quantum yields, depending on the nature of the ligands. For example, in the case of $\left[\mathrm{Ru}(\text { bipy })_{2} \mathrm{~L}(\mathrm{NO})\right]^{2-3+}$ complexes, Da Silva et al. reported $\phi_{\mathrm{NO}}$ value of 0.98 when $\mathrm{L}=\mathrm{Cl}^{-12(\mathrm{~d})}$ This value drops to 0.17 with $\mathrm{L}=4$-picoline and furthermore to 0.07 with $\mathrm{L}$ ) 4-acetylpyridine. ${ }^{12(\mathrm{c})} \mathrm{In}$ $[\mathrm{Ru}(\text { terpy }) \mathrm{L}(\mathrm{NO})]^{3+}$ complexes, $\phi_{\mathrm{NO}}=0.14$ when $\mathrm{L}=$ bipy, but rises to 0.46 when $\mathrm{L}$ is an orthophenylenediamine. ${ }^{17}$ The authors relate these evolutions to the withdrawing character of the $\mathrm{L}$ ligand, which hampered an efficient $\mathrm{Ru} \rightarrow \mathrm{NO}$ charge transfer, assuming that this charge transfer is the dominant parameter to account for the NO· release.

Clearly ruthenium-nitrosyl complexes with polypyridyl ligands possess a very broad range of possible $\phi_{\mathrm{NO}}$ values. However, the mechanism responsible for the release process was never unambiguously established. According to theoretical analyses by Mascharak, the release is ascribed in any case to the population of an excited state having strong $d_{\mathrm{Ru}}-\pi_{\mathrm{NO}} *$ antibonding character. ${ }^{3 \mathrm{a},}{ }^{36}$ Interestingly, the associated transitions tend towards the overall process depicted in equation (1), which leads to: $(i)$ electron transfer to $\mathrm{NO}^{+}$which is switched to $\mathrm{NO}$, (ii) weakening of the Ru-NO bond and finally, (iii) NO· release supporting a mechanism in one step. Apart from this simple picture, different dissociation mechanisms have been suggested with a first excitation towards an additional ligand, however still followed by conversion to the antibonding $d_{\mathrm{Ru}}-\pi_{\mathrm{NO}} *$ excited state. ${ }^{36-38}$ Recently, molecular dynamics beyond the Born-Oppenheimer approximation has been used to point out the possibility for intersystem crossing towards a triplet state susceptible to undergoes additional excitation towards higher triplet states or isomerization to a metastable bent conformation prior to the NO- releasing. ${ }^{9,39}$ The use of multiconfigurational wave functions has also been proposed to provide a better description of the bonding properties of $\mathrm{Ru}(\mathrm{NO})$ metal complexes. ${ }^{40}$ These proposed mechanisms should further be tested by experimental data to support the computational suggestions, although it is difficult to compare experiments conducted on different species at different wavelength irradiations, owing to the number of potential parameters to be taken into account.

A recent investigation in our group has come to the conclusion that the quantum yield of photo-release $\left(\phi_{\mathrm{NO}}\right)$ is hardly correlated with the donating capabilities of the ligands, along a series of substituted $\left[\mathrm{Ru}\left(\mathrm{R} \text {-terpy) } \mathrm{Cl}_{2}(\mathrm{NO})\right]^{+}\right.$complexes. ${ }^{41}$ However, the irradiations were performed at a constant wavelength on these complexes in which the absorption maxima, the nature and the number of the involved transitions were variable, thus hampering reliable conclusions to be established. By contrast, we present here an evaluation of $\phi_{\mathrm{NO}}$ at $\lambda=436 \mathrm{~nm}$ in $[\mathrm{RuT} 1 \mathrm{~B} 0]\left(\mathrm{PF}_{6}\right)_{3}$ and $[\mathrm{RuT} 1 \mathrm{~B} 2]\left(\mathrm{PF}_{6}\right)_{3}$, which undergo the same transition at this wavelength, selected among our set of filters available as the most 
suitable for this investigation. First, the deconvolution of the UV-visible spectra in the low energy domain (Figure 4), is examined to verify the effect of the irradiation on the different species. The wavelengths of the de-convoluted transitions are compared to the TD-DFT data in Table 3, to verify the reliability of the analysis. In the case of $[\mathrm{RuT} 1 \mathrm{~B} 0]\left(\mathrm{PF}_{6}\right)_{3}$, the transition at $425 \mathrm{~nm}$, is the only one active under irradiation at $436 \mathrm{~nm}$. By contrast, the deconvolution reveals the presence of three transitions $(\lambda=340 \mathrm{~nm}, 369 \mathrm{~nm}$, and $421 \mathrm{~nm})$ in closer proximity in [RuT1B2] $\left(\mathrm{PF}_{6}\right)_{3}$, so the resulting shape shows a shoulder at the irradiation wavelength. Nevertheless, it appears that about $90 \%$ of the light provided at $436 \mathrm{~nm}$ is used to promote the transition at $421 \mathrm{~nm}$ (Figure 4, bottom). It can therefore be concluded that, to a large extent, the behaviors of $[\mathrm{RuT1B} 2]\left(\mathrm{PF}_{6}\right)_{3}$ and $[\mathrm{RuT} 1 \mathrm{~B} 0]\left(\mathrm{PF}_{6}\right)_{3}$ should be identical under this irradiation. By contrast, the deconvolution clearly reveals that the irradiation has to be conducted around $\lambda=350 \mathrm{~nm}$ in $[\mathrm{RuT0B} 2]\left(\mathrm{PF}_{6}\right)_{3}$, on the only intense transition observed at low-energy. More precisely, $\lambda=365 \mathrm{~nm}$ was selected as corresponding to the most suitable wavelength in our set of filters available.
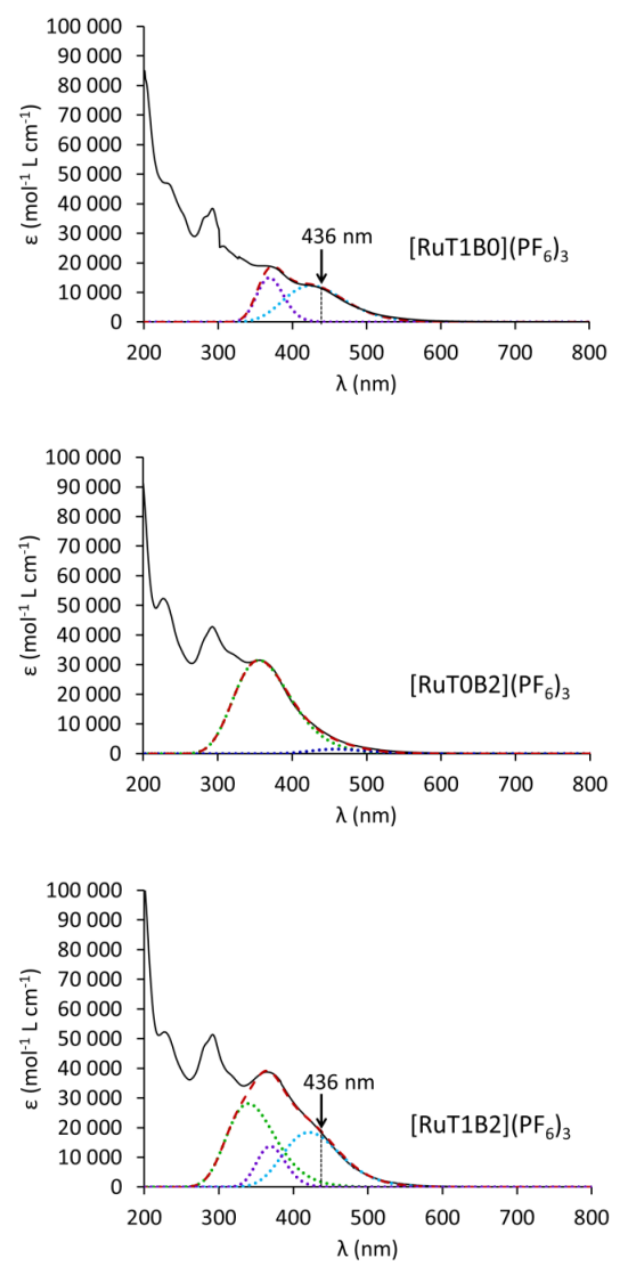

Figure 4 UV-visible spectra of [RuT1B0] $\left(\mathrm{PF}_{6}\right)_{3}$, $[\mathrm{RuT0B} 2]\left(\mathrm{PF}_{6}\right)_{3}$, and $[\mathrm{RuT} 1 \mathrm{~B} 2]\left(\mathrm{PF}_{6}\right)_{3}$ (black solid line) with deconvolutions: the red dashed lines are the sum of the contribution of dotted lines (see text). 
The change in the electronic absorption spectrum of [RuT1B2] $\left(\mathrm{PF}_{6}\right)_{3}$ in acetonitrile, under irradiation at $\lambda=436 \mathrm{~nm}$, is shown in Figure 5. It reveals the presence of isosbestic points at $\lambda=222,266,348$ and $442 \mathrm{~nm}$, which indicate a clean conversion of the $\mathrm{Ru}^{\mathrm{II}}\left(\mathrm{NO}^{+}\right)$complexes to the related photolysed species. No back-reaction is observed when the light is turned off. In the resulting photo-product, new bands arise at 282, 312, and $476 \mathrm{~nm}$. Similar experiments were conducted on [RuT1B0] $\left(\mathrm{PF}_{6}\right)_{3}$ at 436 $\mathrm{nm}$ and on [RuT0B2] $\left(\mathrm{PF}_{6}\right)_{3}$ at $365 \mathrm{~nm}$ (see Supplementary Information). Moreover, the three photoproducts exhibit rather similar UV-visible spectroscopic features with a transition in the 450-500 $\mathrm{nm}$ domain and a multi-transitions band located between 250 and $350 \mathrm{~nm}$.

Table 3 Comparison of the de-convoluted (left) and computed (right) transitions showing the matching between the UV-visible spectrum of $[\mathrm{RuT1B} 2]\left(\mathrm{PF}_{6}\right)_{3}$ and those of the parents $[\mathrm{RuT} 1 \mathrm{~B} 0]\left(\mathrm{PF}_{6}\right)_{3}$ (blue) and $[\mathrm{RuT0B} 2]\left(\mathrm{PF}_{6}\right)_{3}$ (red) compounds.

\begin{tabular}{|c|c|c|c|}
\hline \multicolumn{2}{|l|}{ Experimental data } & \multicolumn{2}{|c|}{ TD-DFT computation } \\
\hline Compound & $\begin{array}{l}\text { deconvoluted } \\
\text { transition }\end{array}$ & Compound & transition \\
\hline$[\mathrm{RuT1B} 0]\left(\mathrm{PF}_{6}\right)_{3}$ & $\begin{array}{l}425 \mathrm{~nm} \\
369 \mathrm{~nm}\end{array}$ & {$[\mathrm{RuT1B} 0]^{3+}$} & $\begin{array}{l}417 \mathrm{~nm} \\
355 \mathrm{~nm}\end{array}$ \\
\hline$[\mathrm{RuT0B} 2]\left(\mathrm{PF}_{6}\right)_{3}$ & $\begin{array}{l}458 \mathrm{~nm} \text { (weak) } \\
\mathbf{3 5 6} \mathrm{nm}\end{array}$ & {$[\text { RuT0B2 }]^{3+}$} & $\begin{array}{l}434 \mathrm{~nm} \text { (weak) } \\
\mathbf{3 9 3} \mathrm{nm}\end{array}$ \\
\hline$[\mathrm{RuT1B} 2]\left(\mathrm{PF}_{6}\right)_{3}$ & $\begin{array}{l}421 \mathrm{~nm} \\
369 \mathrm{~nm} \\
340 \mathrm{~nm}\end{array}$ & {$[\text { RuT1B2 }]^{3+}$} & $\begin{array}{l}413 \mathrm{~nm} \\
382 \mathrm{~nm} \\
349 \mathrm{~nm}\end{array}$ \\
\hline
\end{tabular}

It is worth pointing out that the shape of the UV-visible spectra of the photoproducts (red curve in Figure 5 and Figure S7 in Supplementary Information) does not exhibit the large and broad band centered at $600 \mathrm{~nm}$ characteristic of $\mathrm{Ru}^{\mathrm{III}}$, which is predicted by equation (1). This might suggest that $\mathrm{NO}$ is released as $\mathrm{NO}^{+}$rather than $\mathrm{NO}$. However, this hypothesis has to be abandoned after the examination of the EPR spectra recorded under irradiation in the presence of a $\left[\mathrm{Fe}^{\mathrm{II}}(\mathrm{MGD})_{2}\right]$ which behaves as a powerful spin trapping reagent and leads to the observation of a stable spin adduct exhibiting the characteristic triplet signal of NO (Figure 6). These behaviors are exemplified here for [RuT0B2] $\left(\mathrm{PF}_{6}\right)_{3}$, in Figure 6, with a hyperfine splitting constant value of $\mathrm{a}_{\mathrm{N}}=1.21 \times 10^{-3} \mathrm{~cm}^{-1}$ and a $g$ factor equal to 2.040, values which are fully consistent with reports found in the literature for 
$\left[\mathrm{Fe}^{\mathrm{II}}(\mathrm{MGD})_{2}-\mathrm{NO}\right]$ adducts. ${ }^{23}$ Note that the residual signal observed without irradiation (upper part of Figure 6) is related to the fact that experiments are never strictly conducted in the dark.

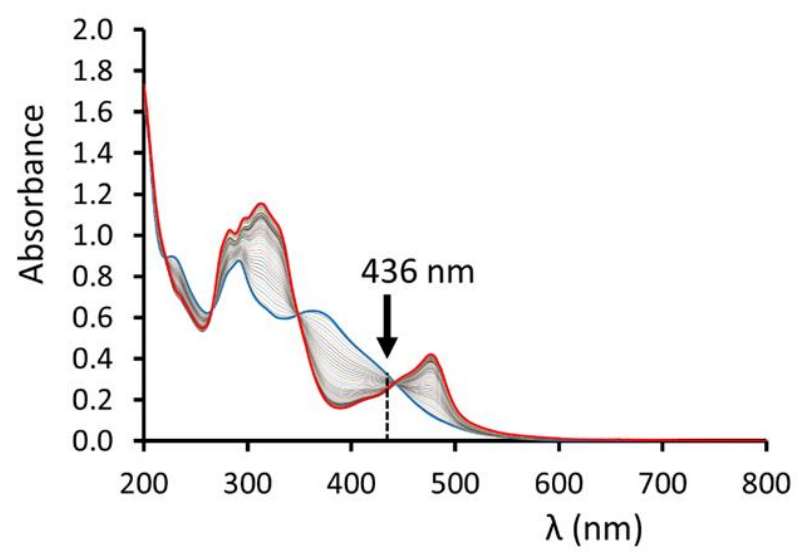

Figure 5 Changes in the absorption spectrum of $\left[\mathrm{RuT}_{1 \mathrm{~B} 2}\right]\left(\mathrm{PF}_{6}\right)_{3}$, in acetonitrile, under irradiation at $\lambda=436 \mathrm{~nm}$. The blue curve corresponds to the starting complex and the red curve corresponds to the photo-product.
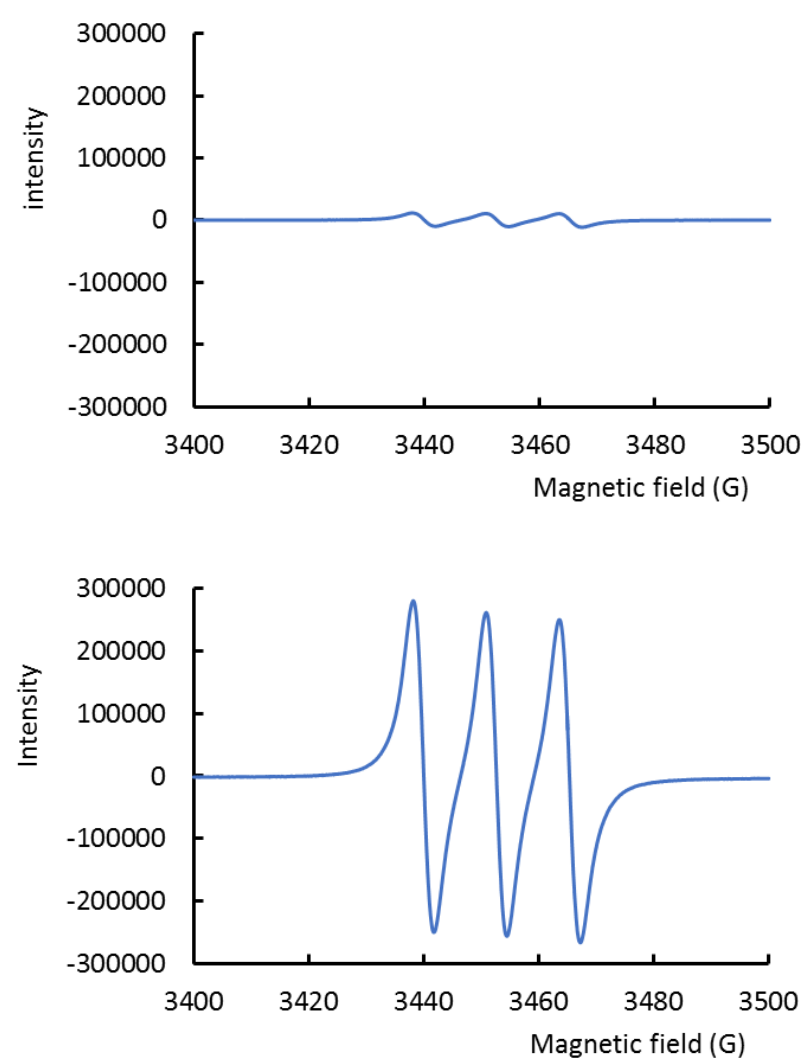

Figure 6 Representative triplet signal from NO trapped by $\left[\mathrm{Fe}^{\mathrm{II}}(\mathrm{MGD})_{2}\right]$ in acetonitrile, after irradiation of [RuT0B2] $\left(\mathrm{PF}_{6}\right)_{3}$, with a Hg lamp (bottom). The signal recorded before irradiation is given as a reference (top). 
Eventually, the observation of a ruthenium (II) complex as the stable final photoproduct is not so surprising. Indeed, we have observed that, after NO· release, the expected $\left[\mathrm{Ru}^{\mathrm{III}} \text { (solvent) }\right]^{3+}$ complex is invariably reduced to a $\mathrm{Ru}^{\mathrm{II}}$, in the case of complexes in which 5 pyridines are present in the coordination sphere, so $\left[\mathrm{Ru}^{\mathrm{II}}\right.$ (solvent)] is the only observable species arising after the NO$\cdot$ release. ${ }^{18} \mathrm{~A}$ previous investigation has pointed out the unexpected appearance of nitrates in the crystal structure of the $\mathrm{Ru}^{\mathrm{II}}$ photoproduct of RuNO complexes containing 5 pyridines. This leads to the suggestion of NO as the potential reducing agent in the fast reduction process. ${ }^{18 \mathrm{c}}$ Indeed, one third of the released NO. available could be used to reduce the photoproducts, as described below:

$$
\begin{aligned}
& \mathrm{Ru}^{\mathrm{III}} \stackrel{+1 e}{\longrightarrow} \mathrm{Ru}^{\mathrm{II}} \\
& 1 / 3 \mathrm{NO} \stackrel{-1 e}{\longrightarrow} 1 / 3 \mathrm{NO}_{3}{ }^{-}
\end{aligned}
$$

No other investigations were carried out to elucidate the nature of the reducing agent accounting for the fast reduction process.

The presence of $\mathrm{Ru}^{\mathrm{II}}$ instead of $\mathrm{Ru}^{\mathrm{III}}$ after irradiation is further confirmed by electrochemistry, with the appearance of an oxidation wave after irradiation along the RuNO series, the values of which are gathered in Table 4. Interestingly, the oxidation becomes more favorable (shifted to lower potentials), as the number of donating substituents increases, in agreement with the chemical intuition. A similar effect is evidenced before irradiation with the $\mathrm{NO}^{+} \rightarrow \mathrm{NO} \cdot$ wave shifted to lower potential indicating that it becomes more difficult to reduce the species having more donating substituents. Altogether the spectroscopic and electrochemical data lead to the conclusion that, although the nature of the reducing agent is not clearly established, Equation (1) has to be re-written as follows:

$$
\left[R u^{I I}\left(N O^{+}\right)\right]+\text {solvent } \stackrel{h v}{\rightarrow}\left[R u^{I I I}(\text { solvent })\right]+N O \stackrel{\text { fast }}{\longrightarrow}\left[R u^{I I}(\text { solvent })\right]+N O \cdot
$$

Finally, the $\phi_{\text {NO }}$ values obtained by treatment of the time dependence of the absorbance of the intense low-energy transitions of $[\mathrm{RuT} 1 \mathrm{~B} 0]\left(\mathrm{PF}_{6}\right)_{3}$, $[\mathrm{RuT0B} 2]\left(\mathrm{PF}_{6}\right)_{3}$, and $[\mathrm{RuT} 1 \mathrm{~B} 2]\left(\mathrm{PF}_{6}\right)_{3}$ are shown in Table 5. Surprisingly, and while the transitions arise from the same general MP $\rightarrow$ RuNO charge transfer behavior in any case, their associated $\phi_{\mathrm{NO}}$ values scale from 0.002 to 0.024 , in a ratio of 1 to 12 . More precisely, the experiments conducted in the same irradiation conditions $(\lambda=436 \mathrm{~nm})$ on [RuT1B2] $\left(\mathrm{PF}_{6}\right)_{3}$ and [RuT1B0] $\left(\mathrm{PF}_{6}\right)_{3}$ lead to $\phi_{\mathrm{NO}}$ values of 0.002 and 0.011 , respectively, in a ratio 1 to 6 contrary to the expectation of unvarying properties arising from identical (MP-terpy $\rightarrow$ RuNO) charge transfer transitions. This striking difference forces us to recognize that the simple one step (transition $\rightarrow$ release) mechanism cannot account for the observation of so different properties. It has therefore to be inferred that the singlet excited state promoted after one-photon absorption cannot release $\mathrm{NO} \cdot$ spontaneously. 
Table 4 Selected redox potential ( $v s$ SCE electrode) observed in the square-wave voltammograms of $[\mathrm{RuT0B} 0]\left(\mathrm{PF}_{6}\right)_{3}, \quad[\mathrm{RuT} 1 \mathrm{~B} 0]\left(\mathrm{PF}_{6}\right)_{3}, \quad[\mathrm{RuT0B} 2]\left(\mathrm{PF}_{6}\right)_{3}, \quad$ and [RuT1B2] $\left(\mathrm{PF}_{6}\right)_{3}$.

\begin{tabular}{lll}
\hline Compound & $\begin{array}{l}\text { before irradiation } \\
\mathrm{NO}^{+} / \mathrm{NO}\end{array}$ & $\begin{array}{l}\text { after irradiation } \\
\mathrm{Ru}^{\mathrm{III}} / \mathrm{Ru}^{\mathrm{II}}\end{array}$ \\
\hline & & \\
{$[\mathrm{RuT0B0}]\left(\mathrm{PF}_{6}\right)_{3}$} & 0.47 & 1.29 \\
{$[\mathrm{RuT1B0}]\left(\mathrm{PF}_{6}\right)_{3}$} & $0.44^{\mathrm{a}}$ & $1.27^{\mathrm{a}}$ \\
{$[\mathrm{RuT0B} 2]\left(\mathrm{PF}_{6}\right)_{3}$} & 0.42 & 1.23 \\
{$[\mathrm{RuT1B} 2]\left(\mathrm{PF}_{6}\right)_{3}$} & 0.39 & 1.20 \\
\hline
\end{tabular}

${ }^{a}$ data from ref. $12 \mathrm{c}$.

Alary et al. have recently proposed that, in $\left[\mathrm{Ru}^{\mathrm{II}}(\operatorname{terpy}) \mathrm{Cl}_{2}(\mathrm{NO}]^{+}\right.$complexes, the Ru-NO bond cannot be broken in the singlet excited state, but is rather switched to a triplet state by intersystem crossing reaction. ${ }^{9}$ Furthermore, this triplet could be subjected to an evolution towards a bent Ru-N-O intermediate, so called MS2 state, subjected to additional photon absorption and finally, to the release of nitric oxide. A two-photon mechanism was recently evidenced in the RuNO/RuON photoisomerization of $\left[\mathrm{Ru}(\mathrm{py})_{4} \mathrm{Cl}(\mathrm{NO})\right]\left(\mathrm{PF}_{6}\right)_{2}$, a reaction which frequently competes with the photorelease and implies first the breaking of the Ruthenium-nitrosyl bond. ${ }^{42}$ Owing to this observation, the issue of a possible sequential two-photon mechanism along the present RuNO series cannot be avoided.

Table 5 Quantum yield of NO photo release $\left(\phi_{\mathrm{NO}}\right)$ after irradiation $\left(\lambda_{\text {irr }}\right)$ on the intense lowenergy transition $\left(\lambda_{\max }\right)$ of $[\mathrm{RuT0B} 0]\left(\mathrm{PF}_{6}\right)_{3},[\mathrm{RuT} 1 \mathrm{~B} 0]\left(\mathrm{PF}_{6}\right)_{3}$, [RuT0B2](PF$)_{3}$, and [RuT1B2] $\left(\mathrm{PF}_{6}\right)_{3}$.

\begin{tabular}{llll}
\hline \multicolumn{1}{c}{ compound } & $\lambda_{\max }(\mathrm{nm})$ & $\lambda_{\text {irr }}(\mathrm{nm})$ & $\phi_{\mathrm{NO}}$ \\
\hline$[\mathrm{RuT0B} 0]\left(\mathrm{PF}_{6}\right)_{3}$ & 352 & 365 & 0.086 \\
{$[\mathrm{RuT1B} 0]\left(\mathrm{PF}_{6}\right)_{3}$} & 425 & 436 & 0.011 \\
{$[\mathrm{RuT0B} 2]\left(\mathrm{PF}_{6}\right)_{3}$} & 356 & 365 & 0.024 \\
{$[\mathrm{RuT1B} 2]\left(\mathrm{PF}_{6}\right)_{3}$} & 421 & 436 & 0.002 \\
\hline
\end{tabular}


To the best of our knowledge, the requirement for a second photon and the need of a triplet state mechanism were never proven experimentally, in the release process. In the present case, the requirement for an intermediate (e.g. MS2) species is not verified experimentally, due to the presence of isosbestic points in Figure 5 which favors a simple two-species $(\mathrm{A} \rightarrow \mathrm{B})$ mechanism. If it is involved here, MS2 must necessarily be extremely photo-reactive, so its concentration stays very weak all over the release process. Although this possibility cannot be strictly dismissed, we clearly have no experimental data in hands suggesting that this two-photon mechanism takes place in our compounds.

As a final comment, it is worth pointing out that the photoproduct generated under irradiation of [RuT1B2] $\left(\mathrm{PF}_{6}\right)_{3}$ undergoes a significant absorption at $436 \mathrm{~nm}$ (Figure 5). As a consequence the completion of the photo-release process required a long irradiation time of about 40 hours. Under these conditions, the issue of possible additional photoreactions of the photoproduced with dissociation of the chelating ligands could have been considered. Indeed, release of bidentate $\pi$ acceptors ligands, such as bipyridine, has been observed under irradiations in some cases. ${ }^{43-46}$ Nevertheless, a set of experimental features allow us to exclude this possibility as a significant behavior: (i) isosbestic point in the UV-vis spectra revealing the presence of a single photoproduct; (ii) photochemical analysis applied satisfactory on the basis of a single photoproduct, (iii) lack of NO in the photoproduct confirmed by EPR and IR spectroscopy (, and (iv) finally presence of a single photoproduct monitored by ${ }^{1} \mathrm{H}$ NMR spectroscopy.

\section{Conclusion}

The report of the new $[\mathrm{RuT0B} 2]\left(\mathrm{PF}_{6}\right)_{3}$, and $\left([\mathrm{RuT} 1 \mathrm{~B} 2]\left(\mathrm{PF}_{6}\right)_{3}\right.$ compounds allowed us to obtain a series of four ruthenium nitrosyl complexes built up from the $\left[\mathrm{Ru}(\text { terpy)(bipy)(NO) }]^{3+}\right.$ core with $0,1,2$, and 3 methoxyphenyl donating units. A computational DFT analysis conducted in support of the experimental data leads to a comprehensive report of the role devoted to the charge transfer effects in both ground and excited states with low-energy transitions arising from a methoxyphenyl to ruthenium nitrosyl charge transfer, in any case. Despite these similarities, irradiations performed on these transitions lead to NO photorelease with significantly different quantum yields, in the broad 0.002 0.02 range. More precisely, and due to orbital orthogonality, the low energy transitions are found identical in [RuT1B0] $\left(\mathrm{PF}_{6}\right)_{3}$ and $[\mathrm{RuT} 1 \mathrm{~B} 2]\left(\mathrm{PF}_{6}\right)_{3}$, nevertheless, with different quantum yield in a ratio of 1 to 6 . This unexpected result proves that the intuitive mechanism based on a simple "absorption + release" scheme is inoperative to account for the experimental data. More sophisticated models are required either by introducing very reacting intermediates or intersystem conversions to hypothetical 
triplet states, as suggested by theorist. Additional experimental data are necessary to clarify if more than one photon is required to release $\mathrm{NO} \cdot$ in these species.

Supplementary information: ${ }^{1} \mathrm{H}$ NMR for the $6 \mathrm{Ru}-\mathrm{X}$ ruthenium complexes $\left(\mathrm{X}=\mathrm{Cl}, \mathrm{NO}_{2}, \mathrm{NO}\right)$ with atom labeling, computational detail (optimized structures and UV-visible spectra), electrochemical and photorelease data for $[\mathrm{RuT} 1 \mathrm{~B} 0]\left(\mathrm{PF}_{6}\right)_{3}$ and $[\mathrm{RuT0B} 2]\left(\mathrm{PF}_{6}\right)_{3} \cdot{ }^{1} \mathrm{H}-\mathrm{NMR}$-tracking of NO release from [RuT1B2] $\left(\mathrm{PF}_{6}\right)_{3}$. IR spectrum for [RuT1B2] $\left(\mathrm{PF}_{6}\right)_{3}$ and its photoproduct.

\section{Acknowledgements}

The authors wish to thank Dr Alix Sounia-Saquet (LCC - CNRS) for performing the electrochemical experiments, Lionel Rechignat (LCC-CNRS) for his assistance in EPR experiment, and Dr Isabelle Sasaki (ISM-Bordeaux) for helpful discussions. M.R. thanks the Ministère de l'Enseignement Supérieur et de la Recherche (MESR) for a PhD fellowship.

\section{References}

1 L. J. Ignarro, Nitric oxide biology and pathobiology, Academic Press, San Diego, 1st edn, 2000 .

2 S. Singh and A. K. Gupta, Cancer Chemother. Pharmacol. 2011, 67, 1211-1224.

3 (a) N. L. Fry and P. K. Mascharak, Acc. Chem. Res. 2011, 44, 289-298; (b) M.J. Rose and P.K. Mascharak, Coord. Chem. Rev. 2008, 252, 2093-2114.

4 for recent reports on NO release from ruthenium nitrosyl complexes, see:

(a) M. Guo, H.J. Xiang, Y. Wang, Q.L. Zhang, L. An, S.P. Yang, Y. Ma, Y. Wang, I.G. Liu and Jin-Gang, Chem. Commun. 2017, 53, 3253-3256; (b) G.L.S. Rodrigues and W.R. Rocha, J. Phys. Chem. B 2016, 120, 11821-11833; (c) N. Cacita, B. Possato, C.F.N. da Silva, C.M. Paulo, A.L.B. Formiga, L.M. Bendhack and S. Nikolaou, Inorg. Chim. Acta 2015, 429, 114-121; (d) J.C.M. Pereira, M.L. Souza and D.W. Franco, Eur. J. Inorg. Chem. 2015, 2015, 1005-1011; (e) K. Ghosh, R. Kumar, K. Kumar, A. Ratnam and U.P. Singh, RSC Adv. 2014, 4, 43599-43605; (f) M.S.P. Marchesi, S.A. Cicillini, A.C.L. Prazias, L.M. Bendhack, A.A. Batista and R.S. Silva, Trans. Met. Chem. 2012, 37, 475-479.

5 J.H. Enemark, and R.D. Feltham, Coord. Chem. Rev. 1974, 13, 339-406. 
R.D. Feltham and J.H. Enemark, Topics in Stereochemistry; Geoffroy, G., Ed.; J. Wiley \& Sons: New York, 1981; Vol. 12, p155.

7 G.K. Lahiri and W. Kaim, Dalton Trans. 2010, 39, 4471-4478.

8 A.P. de Lima Batista, A.G.S. de Oliveira-Filho and S.E. Galembeck, Phys. Chem. Chem. Phys. 2017, 19, 13860-13867.

9 J.S. Garcia, F. Alary, M. Boggio-Pasqua, I.M. Dixon and J.-L. Heully, J. Mol. Model. 2016, $22: 284$

10 G.L.S. Rodrigues and W.R. Rocha, J. Phys. Chem. B 2016, 120, 11821-11833.

11 L. Freitag and L. Gonzalez, Inorg. Chem. 2017, 53, 6415-6426.

12 (a) R. Galvao de Lima, M. Gama Sauaia, C. Ferezin, I. Muniz Pepe, N.M. Jose, L.M. Bendhack, Z. Novais da Rocha and R. Santana da Silva, Polyhedron 2007, 26, 4620-4624 ;

(c) M. Gama Sauaia, R. Galvao de Lima, A.C. Tedesco and R. Santana Da Silva, J. Am. Chem. Soc. 2003, 125, 14718-14719; (d) M. Gama Sauaia, F. de Souza Oliveira, A.C. Tedesco and R. Santana Da Silva, Inorg. Chim. Acta 2003, 355, 191-196; (e) V. Togniolo, R. Santana da Silva and A.C. Tedesco, Inorg. Chim. Acta 2001, 316, 7-12.

13 (a) F.O.N. Silva, M.C.L. Candido, A.K.M. Holanda, I.C.N. Diogenes, E.H.S. Sousa and L.G.F. Lopes, J. Inorg. Biochem. 2011, 105, 624-629 ; (b) F.O.N. Silva, S.X.B. Araujo, A.K.M. Holanda, E. Meyer, F.A.M. Sales, I.C.N. Diogenes, I.M.M. Carvalho, I.S. Moreira, and L.G.F. Lopes, Eur. J. Inorg. Chem. 2006, 10, 2020-2026.

14 E.C. Fornari, M.S.P. Marchesi, A.E.H. Machado and S. Nikolaou, Polyhedron 2009, 28, 1121 1126.

15 (a) A.D. Chowdhury, P. De, S.M. Mobin and G.K. Lahiri, RSC Advances 2012, 2, 3437-3446;

(b) P. De, B. Sarkar, S. Maji, A.K. Das, E. Bulak, S.M. Mobin, W. Kaim and G.K. Lahiri, Eur. J. Inorg. Chem. 2009, 18, 2702-2710; (c) S. Maji, B. Sarkar, M. Patra, A.K. Das, S.M. Mobin, W. Kaim and G.K. Lahiri, Inorg. Chem. 2008, 47, 3218-3227.

16 H. Giglmeier, T. Kerscher, P. Kluefers, D. Schaniel and Th. Woike, Dalton Trans. 2009, 42, 9113-9116.

17 R. Galvao de Lima, M. Gama Sauaia, D. Bonaventura, A.C. Tedesco, L.M. Bendhack and R. Santana da Silva, Inorg. Chim. Acta 2006, 359, 2543-2549;

18 (a) A. Enriquez-Cabrera, I. Sasaki, V. Bukhanko, M. Tassé, S. Mallet-Ladeira, P.G. Lacroix, R.M. Barba-Barba, G. Ramos, N. Farfán, Z. Voitenko and I. Malfant, Eur. J. Inorg. Chem. 2017, 1446-1456.

(b) A. Enriquez-Cabrera, P.G. Lacroix, I. Sasaki, S. Malet-Ladeira, N. Farfan, R.M. BarbaBarba, G. Ramos-Ortiz and I. Malfant, Eur. J. Inorg. Chem. 2018, 531-543.

(c) V. Bukhanko, P.G. Lacroix, I. Sasaki, M. Tassé, S. Mallet-Ladeira, Z. Voitenko and I. Malfant, Inorg. Chim. Acta 2018, 482, 195-205. 
(a) W.R. Murphy Jr., K. Takeuchi, M.H. Barley and T.J. Meyer, Inorg. Chem. 1986, 25, 1041-1053.

(b) D.W. Pipes and T.J. Meyer, Inorg. Chem. 1984, 23, 2466-2472.

E.C. Constable , C.E. Housecroft, M. Neuburger, I. Poleschak and M. Zehnder, Polyhedron, 2003, 22, 93-108.

21 Y. Chen, W. Guo, Z. Ye,G. Wang and J. Yuan, Chem. Comm., 2011, 47, 6266-6268.

22 P.A. Adcock, F. Richard Keene, R. S. Smythe and M.R. Snow, Inorg. Chem., 1984, 23, 23362343.

23 A. J. Liu, Q. Duan, J. Wang, Z. Song, X. Qiao and H. Wang, J. Biomed. Opt., 2015, 20(1), 015004-1 - 015004-7.

24 Gaussian 09, Revision D.01, M. J. Frisch, G. W. Trucks, H. B. Schlegel, G. E. Scuseria, M. A. Robb, J. R. Cheeseman, G. Scalmani, V. Barone, B. Mennucci, G. A. Petersson, H. Nakatsuji, M. Caricato, X. Li, H. P. Hratchian, A. F. Izmaylov, J. Bloino, G. Zheng, J. L. Sonnenberg, M. Hada, M. Ehara, K. Toyota, R. Fukuda, J. Hasegawa, M. Ishida, T. Nakajima, Y. Honda, O. Kitao, H. Nakai, T. Vreven, J. A. Montgomery, Jr., J. E. Peralta, F. Ogliaro, M. Bearpark, J. J. Heyd, E. Brothers, K. N. Kudin, V. N. Staroverov, R. Kobayashi, J. Normand, K. Raghavachari, A. Rendell, J. C. Burant, S. S. Iyengar, J. Tomasi, M. Cossi, N. Rega, J. M. Millam, M. Klene, J. E. Knox, J. B. Cross, V. Bakken, C. Adamo, J. Jaramillo, R. Gomperts, R. E. Stratmann, O. Yazyev, A. J. Austin, R. Cammi, C. Pomelli, J. W. Ochterski, R. L. Martin, K. Morokuma, V. G. Zakrzewski, G. A. Voth, P. Salvador, J. J. Dannenberg, S. Dapprich, A. D. Daniels, Ö. Farkas, J. B. Foresman, J. V. Ortiz, J. Cioslowski, and D. J. Fox, Gaussian, Inc., Wallingford CT, 2009.

25 J. Tomasi, B. Mennucci, and R. Cammi, Chem. Rev. 2005, 105, 2999-3093.

26 (a) P. J. Hay and W. R. Wadt, J. Chem. Phys. 1985, 82, 270-283. (b) W. R. Wadt and P. J. Hay, J. Chem. Phys. 1985, 82, 284-298. (c) P. J. Hay and W. R. Wadt, J. Chem. Phys. 1985, 82, 299-310.

27 for example: (a) M. P. Waller, H. Braun, N. Hojdis and M. Bühl, J. Chem. Theory Comput. 2007, 3, 2234-2242. (b) T. Ayed, J. C. Barthelat, B. Tangour, C. Pradère, B. Donnadieu, M. Grellier and S. Sabo-Etienne, Organometallics 2005, 24, 3824-3826. (c) H. Hratchian and M. C. Milleti, J. Mol. Struct. : THEOCHEM 2005, 340, 119-126. (d) D. Huang, W. E. Streib, O. Eisenstein and K. G. Caulton, Organometallics 2000, 19, 1967-1972.

28 P. Hirva, M. Haukka and M. Jaconen, J. Mol. Modell. 2008, 14, 171-181.

29 T. Yanai, D. Tew, and N. Handy, Chem. Phys. Lett. 2004, 393, 51-57.

30 Program Sa3.3 written by D. Lavabre and V. Pimienta (http://cinet.chim.pagespersoorange.fr/tele_sa/install_Sa.html).

31 V. Pimienta, C. Frouté, M.-H. Deniel, D. Lavabre, R. Guglielmetti and J. C. Micheau, J. Photochem. Photobiol. A, 1999, 122, 199-204 (reference 35). 
32 J. Akl, Ch. Billot, P.G. Lacroix, I. Sasaki, S. Malet-Ladeira, I. Malfant, R. Arcos-Ramos, M. Romero and N. Farfan, New J. Chem. 2013, 37, 3518-3527.

33 M. Roose, I. Sasaki, V. Bukhanko, S. Mallet-Ladeira, R.M. Barba-Barba, G. Ramos-Ortiz, A. Enriquez-Cabrera, N. Farfan, P.G. Lacroix and I. Malfant, Polyhedron 2018, 151, 100-111.

34 K. Abbas and D. Marji, Zeitschrift fur Naturforsch. - Sect. A J. Phys. Sci. 2005, 60, 667-671.

35 F. Kanoufi, Y. Zu and A.J. Bard, J. Phys. Chem. B 2001, 105, 210-216.

36 N.L. Fry and P.K. Mascharak, Dalton Trans. 2012, 41, 4726-4735.

37 A.C. Merkle, N.L. Fry, P.K. Mascharak and N. Lehnert, Inorg. Chem. 2011, 50, 12192-21203

38 W. Zheng, S. Wu, S. Zhao, Y. Geng, J. Jin, Z. Su and Q. Fu, Inorg. Chem. 2012, 51, 39723980.

39 L. Freitag and L. González, Inorg. Chem. 2014, 53, 6415-6426

40 A.P. de Lima Batista, A.G.S. de Oliveira-Filho, and S.E. Galembeck, Phys. Chem. Chem. Phys. 2017, 19, 13860-13867.

41 S. Amabilino, M. Tasse, P.G. Lacroix, S. Mallet-Ladeira, V . Pimienta, J. Akl, I. Sasaki and I. Malfant, New J. Chem. 2017, 41, 7371-7383.

42 L. Khadeeva, W. Kaszub, M. Lorenc, I. Malfant and M. Buron-Le Cointe, Inorg.Chem. 2016, 55, 4117-4123.

43 L.M. Loftus, K.F. Al-Afyouni, Kathlyn F. and C. Turro, Chem Eur. J. 2018, 24, 11550-11553.

44 D. Havrylyuk, D.K. Heidary, L. Nease, S. Parkin and E.C. Glazer, Eur. J. Inorg. Chem. 2017, 1687-1694.

45 E. Wachter, D.K. Heidary, B.S. Howerton, S. Parkin and E.C. Glazer, Chem. Commun. 2012, 48, 9649-9651

46 A.C. Laemmel, J.P. Collin and J.P. Sauvage, Eur. J. Inorg. Chem. 1999, 383-386. 